\title{
Depletion of Arg/Abl2 improves endothelial cell adhesion and prevents vascular leak during inflammation
}

\author{
Joana Amado-Azevedo ${ }^{1} \cdot$ Anne-Marieke D. van Stalborch ${ }^{2} \cdot$ Erik T. Valent $^{1} \cdot$ Kalim Nawaz $^{2} \cdot$ Jan van Bezu $^{1}$. \\ Etto C. Eringa ${ }^{1}$ - Femke P. M. Hoevenaars ${ }^{1}$. Iris M. De Cuyper ${ }^{2}$. Peter L. Hordijk ${ }^{1}$ - Victor W. M. van Hinsbergh ${ }^{1}$. \\ Geerten P. van Nieuw Amerongen ${ }^{1}$. Jurjan Aman ${ }^{1,3}$. Coert Margadant ${ }^{4}$
}

Received: 21 October 2020 / Accepted: 6 March 2021 / Published online: 26 March 2021

(c) The Author(s) 2021, corrected publication 2021

\begin{abstract}
Endothelial barrier disruption and vascular leak importantly contribute to organ dysfunction and mortality during inflammatory conditions like sepsis and acute respiratory distress syndrome. We identified the kinase Arg/Abl2 as a mediator of endothelial barrier disruption, but the role of Arg in endothelial monolayer regulation and its relevance in vivo remain poorly understood. Here we show that depletion of Arg in endothelial cells results in the activation of both RhoA and Rac1, increased cell spreading and elongation, redistribution of integrin-dependent cell-matrix adhesions to the cell periphery, and improved adhesion to the extracellular matrix. We further show that Arg is activated in the endothelium during inflammation, both in murine lungs exposed to barrier-disruptive agents, and in pulmonary microvessels of septic patients. Importantly, Argdepleted endothelial cells were less sensitive to barrier-disruptive agents. Despite the formation of F-actin stress fibers and myosin light chain phosphorylation, Arg depletion diminished adherens junction disruption and intercellular gap formation, by reducing the disassembly of cell-matrix adhesions and cell retraction. In vivo, genetic deletion of Arg diminished vascular leak in the skin and lungs, in the presence of a normal immune response. Together, our data indicate that Arg is a central and non-redundant regulator of endothelial barrier integrity, which contributes to cell retraction and gap formation by increasing the dynamics of adherens junctions and cell-matrix adhesions in a Rho GTPase-dependent fashion. Therapeutic inhibition of Arg may provide a suitable strategy for the treatment of a variety of clinical conditions characterized by vascular leak.
\end{abstract}

Keywords Arg/Abl2 $\cdot$ Endothelial barrier function · Inflammation · Integrins $\cdot$ Vascular leak $\cdot$ VE-cadherin

Joana Amado-Azevedo, Anne-Marieke D. van Stalborch, Erik T.

Valent have contributed equally to this work (shared first author),

Jurjan Aman and Coert Margadant have contributed equally to this work (shared last author).

Jurjan Aman

j.aman@amsterdamumc.nl

1 Department of Physiology, Amsterdam Cardiovascular Sciences, Amsterdam University Medical Center,

Amsterdam, The Netherlands

2 Sanquin Research, Amsterdam, The Netherlands

3 Department of Pulmonology, Amsterdam Cardiovascular Sciences, Amsterdam University Medical Center,

Amsterdam, The Netherlands

4 Angiogenesis Laboratory, Department of Medical Oncology, Cancer Center Amsterdam, Amsterdam University Medical Center, Amsterdam, The Netherlands

$\begin{array}{ll}\text { Abbreviations } \\ \text { AJ } & \text { Adherens junction } \\ \text { ARDS } & \text { Acute respiratory distress syndrome } \\ \text { Arg } & \text { Abl-related gene } \\ \text { CrkL } & \text { Crk-like } \\ \text { EB } & \text { Evans blue } \\ \text { ECIS } & \text { Electrical cell-substrate impedance sensing } \\ \text { ECM } & \text { Extracellular matrix } \\ \text { FA } & \text { Focal adhesion } \\ \text { FB } & \text { Fibrillar adhesion } \\ \text { FN } & \text { Fibronectin } \\ \text { HSA } & \text { Human serum albumin } \\ \text { HPMVEC } & \text { Human pulmonary microvascular endothe- } \\ & \text { lial cell } \\ \text { HUVEC } & \text { Human umbilical vein endothelial cell } \\ \text { LPS } & \text { Lipopolysaccharide } \\ \text { MLC } & \text { Myosin light chain } \\ \text { pMLC } & \text { Phospho-MLC } \\ \text { P(Y) } & \text { Phosphotyrosine }\end{array}$


VE-cadherin Vascular endothelial cadherin

VEGF Vascular endothelial growth factor WT Wild type

\section{Introduction}

Endothelial barrier disruption and vascular leak are hallmarks of a variety of life-threatening conditions including sepsis, acute respiratory distress syndrome (ARDS), and COVID-19, and importantly contribute to the morbidity and mortality of critically ill patients [1-3]. Despite the serious adverse clinical outcome associated with vascular leak, no treatment is currently available to reverse endothelial barrier disruption [2, 4].

Barrier function critically depends on intercellular adhesion complexes including adherens junctions (AJs), formed by vascular endothelial (VE)-cadherin. In addition, barrier function requires cell adhesion to the basement membrane maintained by integrins, a family of $\alpha \beta$ heterodimeric transmembrane receptors crucial for the assembly of cytoskeleton-associated adhesion complexes such as focal adhesions (FAs) [4]. During injury or inflammation, endothelial barrier disruption is induced by a variety of agents including lipopolysaccharide (LPS), inflammatory cytokines, or thrombin, which trigger a rapid and transient increase in permeability by activation of the small GTPase RhoA, leading to a Rho kinase-dependent increase in myosin light chain (MLC) phosphorylation and the formation of actin stress fibers [5-9]. The resulting cytoskeletal contractility transforms stable AJs into remodeling junctions, which is associated with VE-cadherin internalization, thus promoting cell retraction and increasing endothelial permeability [7-10]. This process is counteracted by cell spreading dependent on the GTPase Rac1, which promotes the assembly of peripheral cell-matrix adhesions and AJs [7, 11-13]. Thus, a tight balance between Rac1 and RhoA activities regulates cell spreading versus contractility, and is essential for normal barrier regulation.

Extensive crosstalk exists between integrins, Rho GTPases, and cell-cell adhesions. Vascular endothelial cells express several integrins of the $\beta 1$ subfamily that bind to ligands in the extracellular matrix, which reinforces VEcadherin-dependent cell-cell adhesion and contributes to barrier function in quiescent endothelium [14-16]. Furthermore, regulators of integrins such as talin, which binds integrin $\beta$-cytoplasmic tails to stimulate integrin activation, also reinforce AJs and are essential for endothelial barrier function [17]. However, in recent years it has become clear that $\beta 1$ integrins, in particular $\alpha 5 \beta 1$, can also promote barrier disruption in pathological conditions such as inflammation $[18,19]$. Integrin $\alpha 5 \beta 1$ binds to fibronectin $(\mathrm{FN})$, which is strongly upregulated during injury, remodeling, and inflammation $[20,21]$. The interaction between FN and $\alpha 5 \beta 1$ induces robust RhoA activation, which in turn triggers the assembly of FN fibrils and associated fibrillar adhesions (FBs), a special kind of tension-bearing adhesion complexes located in the cell center that facilitate cytoskeletal contractility and cell contraction [22-24]. Indeed, we and others have previously found that $\alpha 5 \beta 1$ disrupts cadherindependent cell-cell junctions through RhoA-dependent contractility, in a variety of cell types including endothelial cells $[18,19,25,26]$. Furthermore, blocking $\beta 1$ integrins was recently shown to protect from LPS-induced barrier disruption and vascular leakage in mice $[18,19]$. Thus, integrins exert dual functions on endothelial barrier function, through their effects on Rho GTPases and crosstalk with cell-cell junctions.

We have previously found in pre-clinical studies that the tyrosine kinase inhibitor imatinib (Gleevec ${ }^{\circledR}$ ) preserves endothelial barrier integrity by inhibition of the tyrosine kinase Abl-related gene (Arg/Abl2) [27], corroborating clinical reports which demonstrated that imatinib reverses vascular leak and pulmonary edema in patients [28-31]. These data suggest that Abelson-family kinases are potential targets for the treatment of vascular leak [30], and we have recently completed clinical trials with imatinib to prevent pulmonary vascular leak in patients with respiratory failure due to COVID-19 (EudraCT \# 2020-001236-10).

Imatinib treatment in endothelial and other cells induced an increase in FA numbers in vitro, in particular at the cell periphery, suggesting that the Abelson kinases regulate the organization of cell-matrix adhesions [27, 32]. Furthermore, knockdown of both kinases in epithelial cells revealed that they regulate the stability of AJs, through their effects on Rac and Rho activities [33]. The combined targeted deletion of the genes encoding c-Abl (Abl1) and Arg in mice is embryonically lethal by E9.5, due to disrupted vascular development and endothelial cell apoptosis [34]. Finally, the endothelial-specific deletion of the gene encoding c-Abl in heterozygous $\mathrm{Arg}^{+/-}$mice protects against endothelial barrier disruption [35], in line with other studies showing that c-Abl regulates barrier function [36-38]. However, the specific role of Arg in endothelial barrier regulation is poorly understood and remains unexplored. Intriguingly, studies in fibroblasts have shown that Arg phosphorylates p190RhoGAP, which inhibits RhoA activation and RhoAdependent FA turnover [39, 40]. Other Arg substrates associated with integrin-dependent cell-matrix adhesions include cortactin, Crk and CrkL, as well as the cytoplasmic tail of the integrin $\beta 1$ subunit [41-43].

In this study, we find that Arg is a key regulator of Rho GTPase activation, cell-matrix adhesion, and cell-cell adhesion in endothelial cells. Arg promotes RhoA-dependent cell retraction, VE-cadherin internalization, and endothelial barrier disruption. Depletion of Arg protects against 
barrier disruption in vitro, and Arg-deficient mice are protected from LPS-induced vascular leak and pulmonary edema. Thus, our data suggest that Arg inhibition improves endothelial barrier function, and constitutes a strategy for the treatment of respiratory failure caused by endothelial dysfunction.

\section{Results}

\section{Arg regulates Rac1 activation, cell spreading, and organization of cell-matrix adhesions in endothelial cells}

To investigate the role of Arg in endothelial cells, we depleted Arg from human umbilical vein endothelial cells (HUVECs) by RNA interference. Arg expression was reduced up to $85 \%$ at the mRNA and protein level, as compared to control cells that received scrambled sequences, while c-Abl expression was not significantly downregulated (Suppl. Fig. S1a, b).

Arg depletion induced a number of alterations in HUVEC morphology and the organization and distribution of cellmatrix adhesions. Arg-depleted cells became larger and on average more elongated than control cells (Fig. 1a-c). This phenomenon was observed in monolayers but even more prominently in sparsely seeded cells, thus excluding indirect effects resulting from potential differences in cell number or confluency, and suggesting that integrin- and/ or Rac-dependent cell spreading were enhanced (Suppl. Fig. S2a). In line with this, downregulation of Arg induced increased HUVEC adhesion to the $\beta 1$ integrin ligands Col-1 and FN in adhesion assays (Fig. 1d). Overexpression of Arg has been shown to promote phosphorylation of Y783 in the $\beta 1$ cytoplasmic tail, which is important for talin binding and integrin activation [42]. Therefore, we next assessed the activation status of $\beta 1$, using the antibody 9EG7 that recognizes the high-affinity conformation of $\beta 1$ integrins. The proportion of active $\beta 1$ integrins was unchanged in Arg-depleted HUVECs, but the total amount of $\beta 1$ integrins was slightly increased, in line with the observation that expression of an active Arg mutant reduced $\beta 1$ expression in epithelial cells [44] (Suppl. Fig. S2b, c). Next we examined whether Arg phosphorylates Y783 in the $\beta 1$ cytoplasmic tail in HUVECs, and whether this was affected by thrombin, which promotes RhoA-dependent endothelial barrier disruption [8]. Y783 phosphorylation was only detectable when cells were treated with $\mathrm{Na} 3 \mathrm{VO} 4$ to inhibit phosphatases, and a transient decrease followed by recovery was observed upon thrombin addition (Suppl. Fig. S2d). However, the depletion of Arg had no apparent effect (Suppl. Fig. S2d).

We then assessed whether Arg affects the organization or distribution of cell-matrix adhesions, either by ectopic expression of GFP-tensin-1, a marker for FBs, or using antibody 4G10 that detects phosphorylated tyrosines, a marker for FAs (Fig. 1a). FA formation seemed enhanced in Argdepleted HUVECs, especially at the cell periphery close to VE-cadherin-based cell-cell junctions (Fig. 1a and data not shown), reminiscent of what we have previously described for HUVECs treated with imatinib [27]. Similar results were obtained using antibodies against FA markers paxillin or vinculin (Suppl. Fig. S2a and data not shown). The increase in cell-matrix adhesions and their enrichment at the cell periphery became particularly evident when examining the distribution of integrin $\alpha 5 \beta 1$ (Fig. 1e, f). Finally, we tested whether Arg regulates the activation of Rac1, by determining the active fractions of these GTPases in pulldown assays. Basal Rac1 activity was elevated in Arg-depleted HUVECs, explaining the enhanced cell spreading (Fig. 1g).

Taken together, these data show that Arg is a major regulator of integrin-dependent cell-matrix adhesion and spreading in endothelial cells.

\section{Arg regulates RhoA activation, MLC phosphorylation, and AJ morphology under nonstimulated conditions}

Since there is a tight balance between Rac1 activity/integrin-dependent cell spreading on the one hand, and RhoA activity/actomyosin contraction on the other hand, we next analyzed whether Arg regulates RhoA activity and signaling. By determining the active fractions in pulldown assays, we observed that basal RhoA activation was significantly enhanced upon Arg silencing (Fig. 2a). This result is consistent with previous studies in fibroblasts [39], and also with the observed increase in cell-matrix adhesions (Fig. 1). Furthermore, Arg-depleted HUVECs assembled a more extensive FN fibril network, which requires integrin $\alpha 5 \beta 1$ and RhoA-dependent cytoskeletal contractility (Fig. 2b).

We then assessed the effect of Arg on VE-cadherinbased AJs. The expression of VE-cadherin or $\beta$-catenin was not affected by Arg silencing in HUVECs in basal conditions (Suppl. Fig. S3a). Using $\mathrm{Arg}^{-/-}$versus wild-type (WT) littermates obtained from $\mathrm{Arg}^{+/-} \times \mathrm{Arg}^{+/-}$breedings (Suppl. Fig. S4a), we observed that the expression of VE-cadherin was also unchanged in Arg knockout $\left(\mathrm{Arg}^{-/}\right)$mice (Suppl. Fig. S3b). However, AJs in vessels of $\mathrm{Arg}^{-1-}$ mice appeared 'thinner' and more 'jagged' than those in WT mice (Fig. 2c). A similar phenotype was observed in cultured endothelial cells (Fig. 2d), indicating that AJs experienced increased actomyosin-induced pulling forces, consistent with the observed high basal activation of RhoA in Arg-depleted HUVECs. This was further suggested by increased myosin light chain (MLC) monophosphorylation (pSer19) in Arg-depleted cells under basal conditions, which was observed close to 


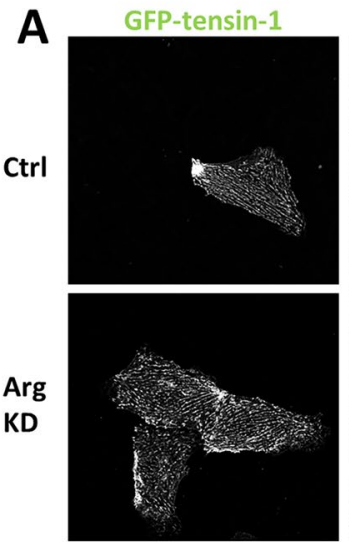

B

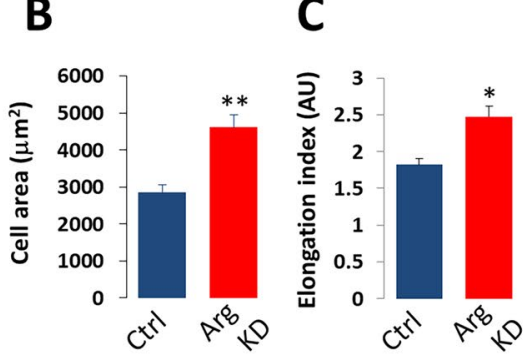

F-actin
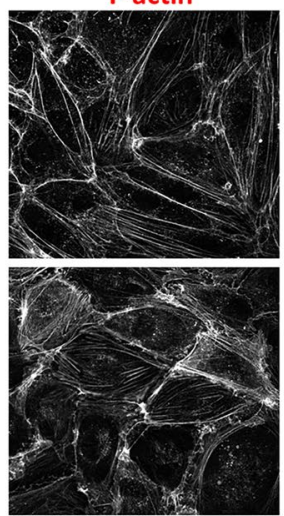

Phosphotyrosines
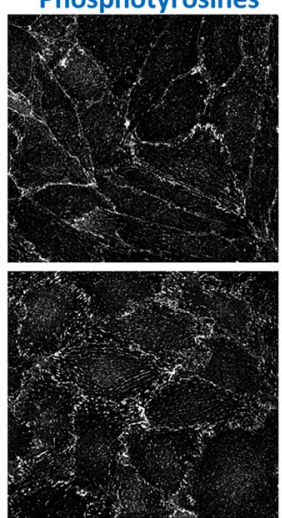

D
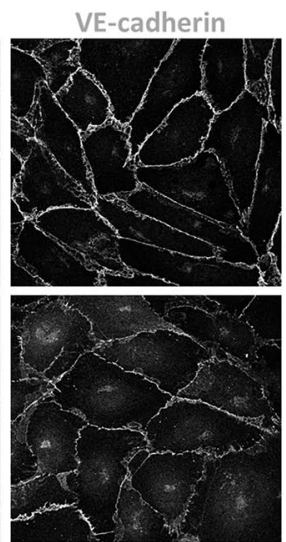

$\mathbf{E}$
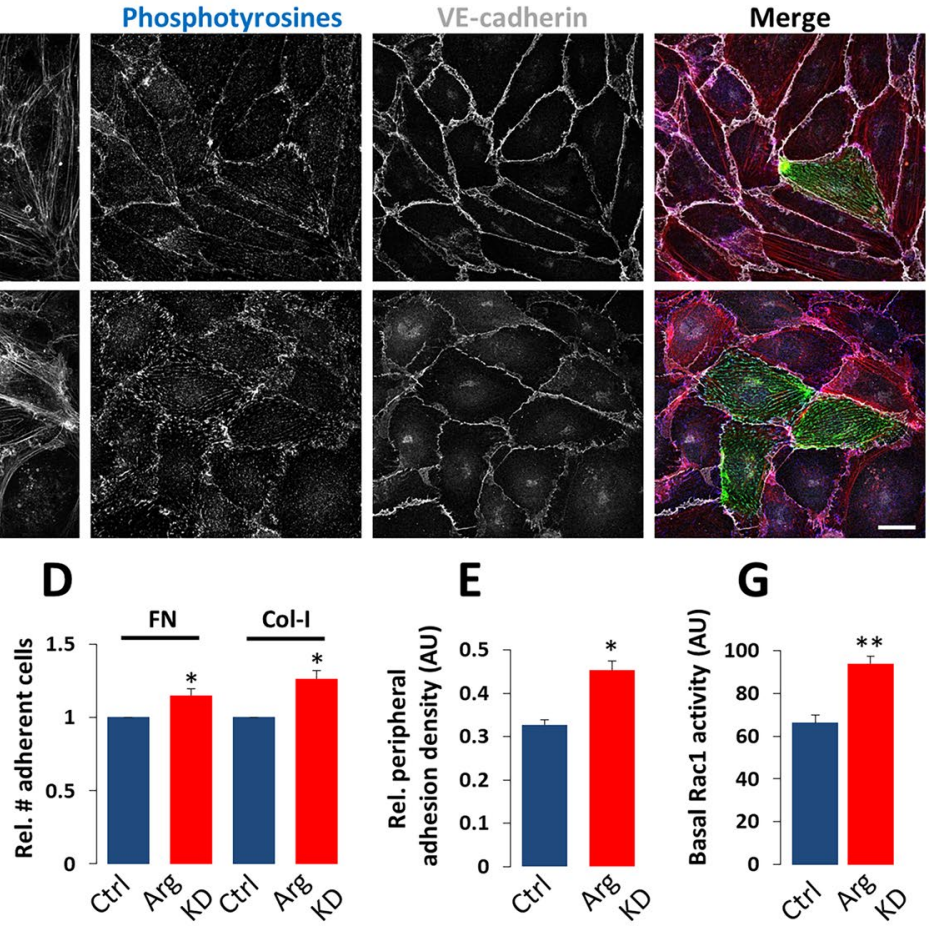

$\mathbf{F}$

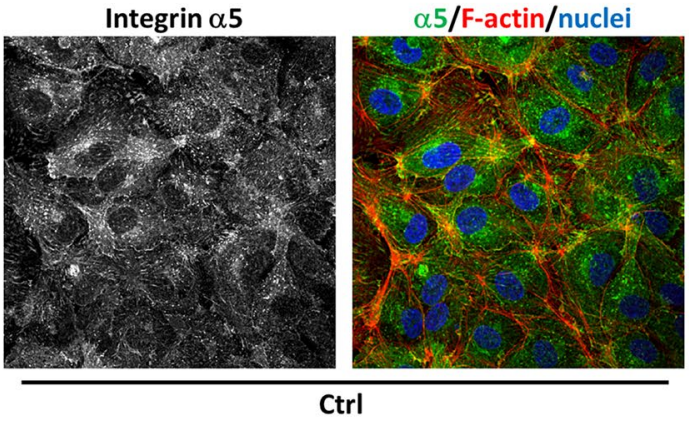

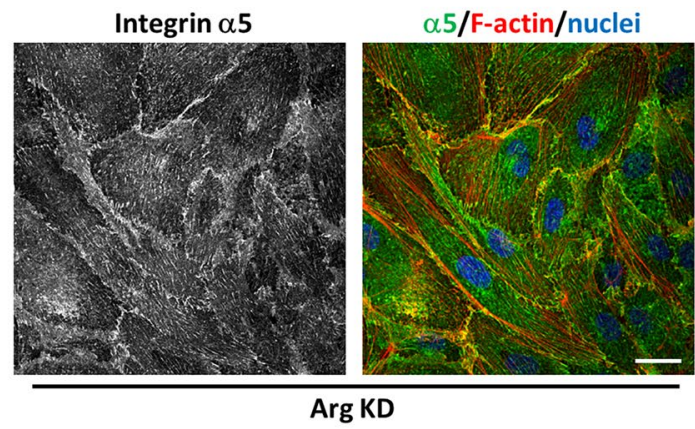

SEM of $n=4$ experiments. e Quantification of peripheral distribution of integrin $\alpha 5$ quantified from confocal images as in (f), as described in Methods. Means + SEM of $n=20$ cells/condition. Rel., relative. f Representative confocal images of integrin $\alpha 5$ (green) and F-actin (red) in control and Arg-depleted HUVEC monolayers. Nuclei are stained with DAPI (blue). Bar, $40 \mu \mathrm{m}$. g Basal Rac1 activity as assessed in pulldown assays. Data are means + SEM from $n=3$. $* \mathrm{P}$ $<0.05 ; * * \mathrm{P}<0.01$. AU, arbitrary units.

Together, these findings show that Arg regulates RhoA activation and signaling in endothelial cells, as well as the organization of AJs.

\section{Arg promotes cell retraction in response to thrombin}

We next investigated how Arg regulates the behavior of endothelial cells when exposed to endothelial barrierdisruptive agents. For this purpose, we chose thrombin, which is a well-studied RhoA activator involved in 
A

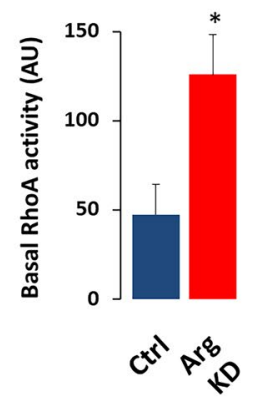

C
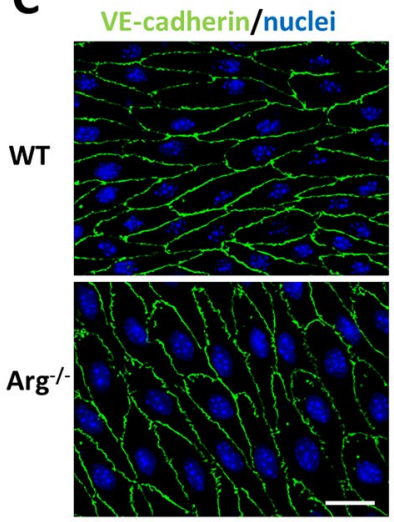

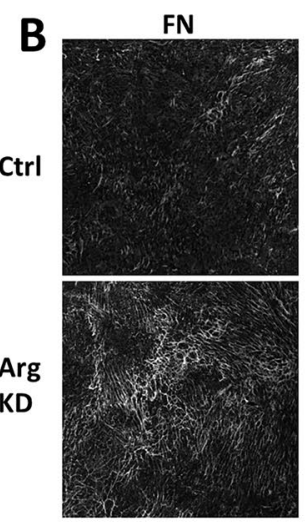

D pMLC

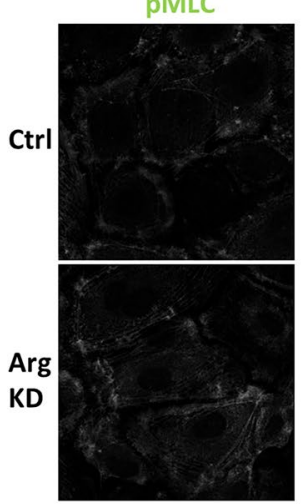

F-actin/nuclei

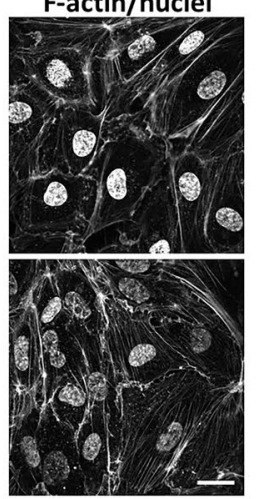

F-actin

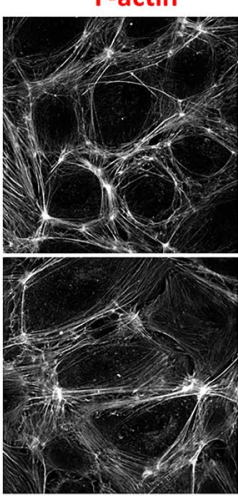

E

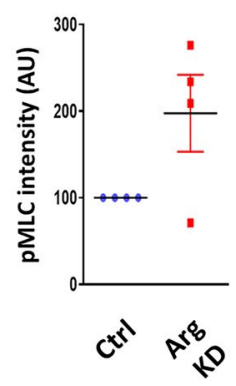

VE-cadherin

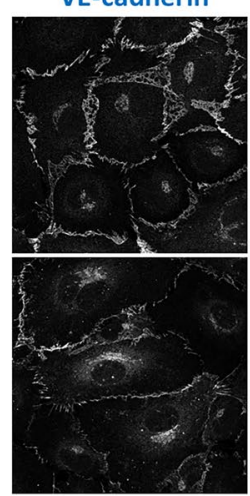

$\mathbf{F}$
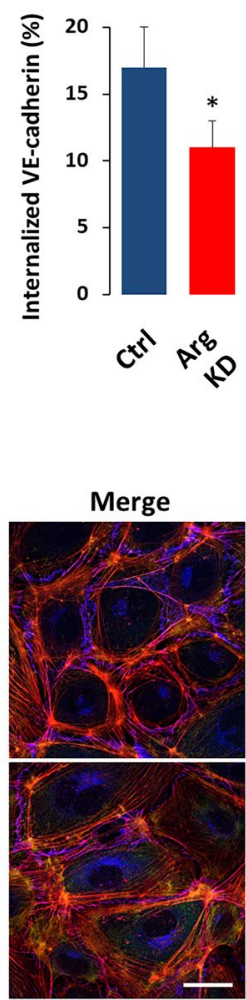

Fig. 2 Arg controls RhoA activation, MLC phosphorylation, and AJ morphology in HUVECs. a Basal RhoA activity was determined in pulldown assays. Data are means + SEM from $n=3$. b Confocal images of FN fibrils (left) and F-actin/DAPI (right) in control and Arg-depleted HUVEC monolayers. Bar, $30 \mu \mathrm{m}$. c Confocal images of VE-cadherin (green) and nuclei (blue) in femoral arteries of WT and

endothelial barrier disruption. Treatment with thrombin for up to $30 \mathrm{~min}$ potently increased RhoA activation in control HUVECs, confirming many other studies $[9,11$, 13] (Fig. 3a and data not shown). However, the thrombininduced increase in RhoA activation was significantly lower in Arg-depleted cells, probably because RhoA activity was already high in basal conditions (Fig. 3a). Rac1 activity was slightly but not significantly increased by thrombin in this time-frame, and was higher in Argdepleted cells both in the absence and the presence of thrombin (Fig. 3b). In line with the observed differences in RhoA activation, thrombin induced a strong increase in MLC phosphorylation and FN fibrillogenesis in control cells, while Arg-depleted cells had already high levels of phospho-MLC and FN fibrils under basal conditions (Fig. 3c-e). Thrombin-induced increase in phospho-MLC was observed on actin stress fibers and along the entire cell periphery, in particular on circumferential actin filaments (Fig. 3e).
$\mathrm{Arg}^{-1-}$ mice. Bar, $10 \mu \mathrm{m}$. d Representative confocal images of pMLC (green), F-actin (red), and VE-cadherin (blue) in control and Argdepleted HUVEC monolayers. Bar, $30 \mu \mathrm{m}$. e Relative pMLC intensity quantified from confocal images. $A U$ arbitrary units. f Internalization of VE-cadherin in control and Arg-depleted HUVEC monolayers. Data are means $+\operatorname{SEM}$ from $n=3$. $* \mathrm{P}<0.05$

Morphologically, thrombin-induced cell contraction and intercellular gap formation (indicated by asterisks in Fig. 3f) was evident in control, but not in Arg-depleted HUVECs, in which cell spreading remained better preserved (Fig. 3f). The latter appeared not to be due to differences in stress fiber formation (Fig. 3e, f). Arg-depleted cells retained peripheral FAs during thrombin stimulation, as well as centrally located FBs, whereas in control cells a strong, polarized accumulation of tensin was observed, presumably due to RhoAdependent FB redistribution (indicated by arrows in Fig. 3f).

To analyze the dynamic behavior of FBs in live cells, HUVECs were transfected with GFP-tensin-1, grown to confluency, and the response to thrombin was analyzed by time-lapse microscopy. While thrombin induced clear sliding, accumulation, and eventual disappearance of FBs in control cells, FBs in shArg cells seemed much more resistant against this effect (data not shown). Quantification of the assembly and disassembly rate revealed that whereas the assembly rate was comparable, the disassembly rate of 
A

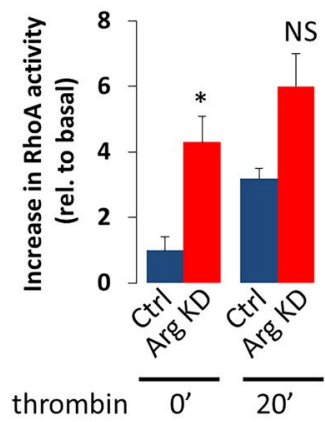

B

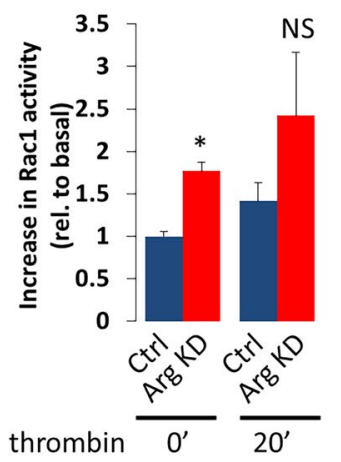

C
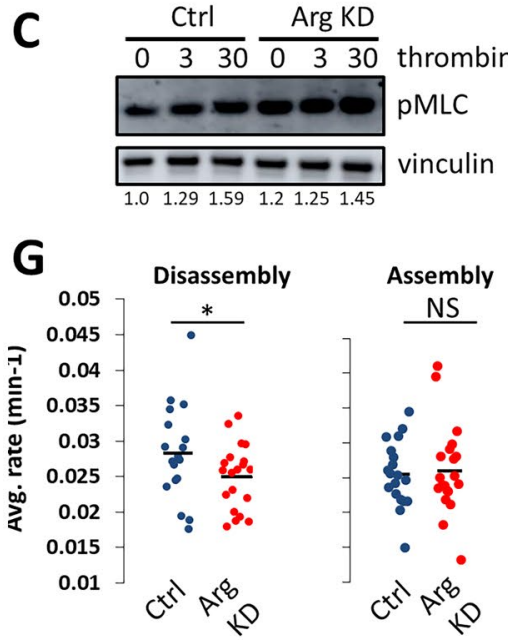

D
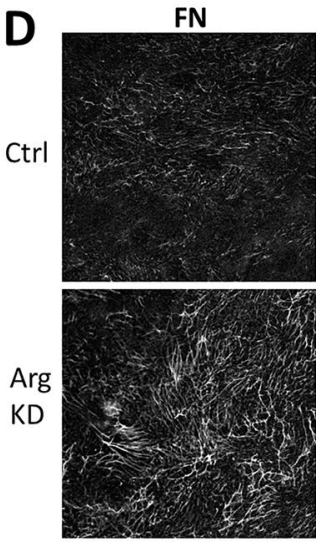

F-actin/nuclei

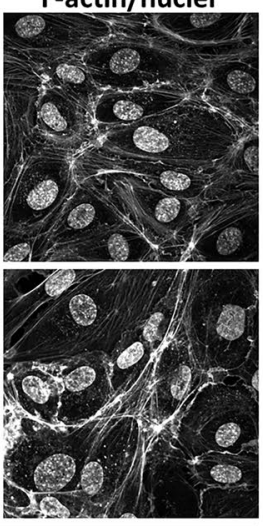

$0^{\prime}$ thrombin
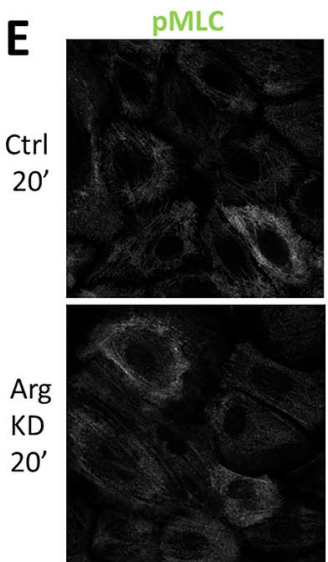

F
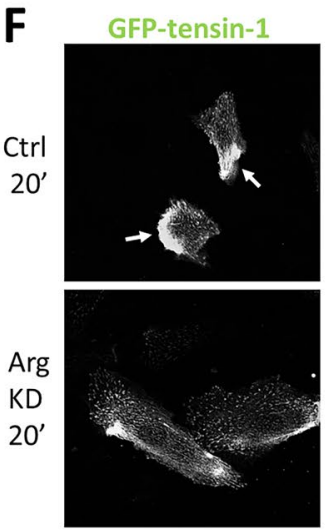

F-actin

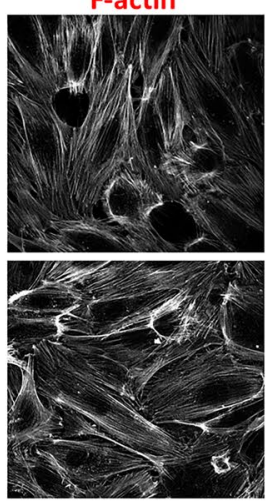

FN

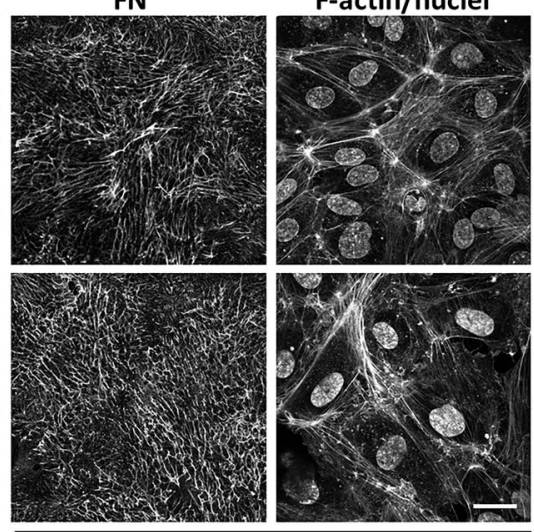

$20^{\prime}$ thrombin

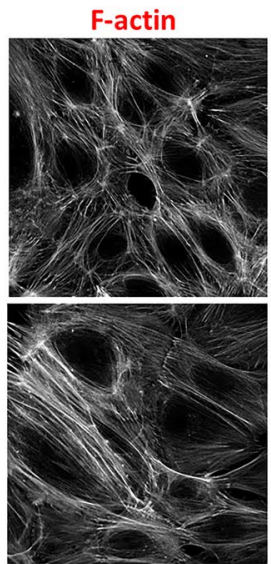

VE-cadherin

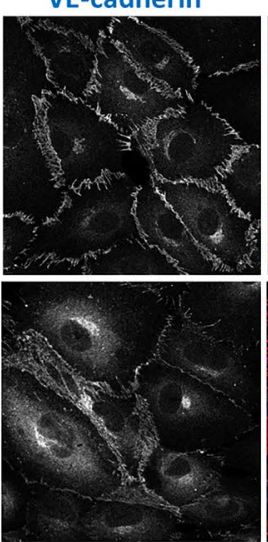

Phosphotyrosines
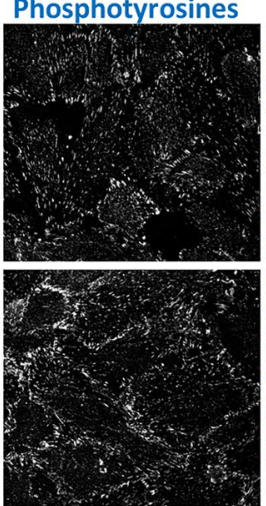

Merge

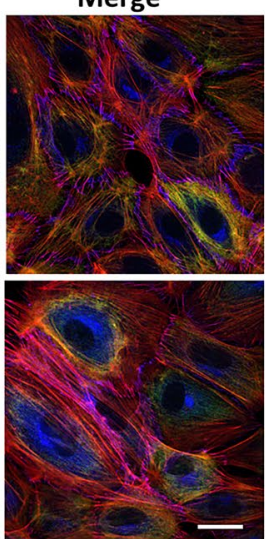

Merge

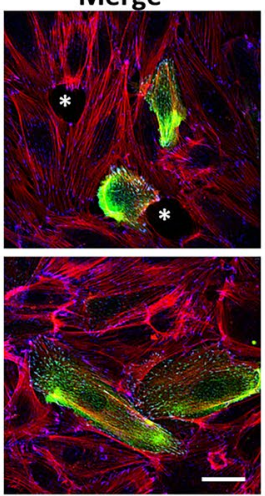

Fig. 3 Arg enhances thrombin-induced RhoA activation, redistribution of cell-matrix adhesions, and cell retraction in HUVECs. a RhoA activity was determined in pulldown assays at 0 and 20 mins of thrombin stimulation. Data are means + SEM from $n=3$. b Rac1 activity at 0 and 20 mins of thrombin stimulation. Data are means + SEM from $n=3$. c MLC phosphorylation in control and Arg-depleted HUVEC monolayers at the indicated time-points after thrombin addition. Numbers indicate normalized amounts of pMLC, quantified by densitometry and expressed relative to the amount in Ctrl cells at $\mathrm{t}=0$. d Confocal images of FN fibrils and F-actin/DAPI in control (top) and Arg-depleted HUVEC (bottom) monolayers at 0 and 20 mins of thrombin stimulation. Bar, $30 \mu \mathrm{m}$. e Confocal images of pMLC (green), F-actin (red), and VE-cadherin (blue) in control and Arg-depleted HUVEC monolayers. Bar, $30 \mu \mathrm{m}$. f Representative confocal images showing the distribution of GFP-tensin-1 (green), F-actin (red), and phosphotyrosines (blue) in control (top) and Argdepleted (bottom) HUVEC monolayers 20 mins after thrombin stimulation. Arrows indicate GFP-tensin-1 accumulation, asterisks denote intercellular gaps. Bar, $40 \mu \mathrm{m}$. g Average assembly and disassembly rate of FBs in control and Arg-depleted HUVECs in thrombin-stimulated monolayers. Each data point represents 1 time-lapse movie. NS, not significant; $* \mathrm{P}<0.05$ 
FBs was indeed significantly lower upon Arg silencing, thus reducing their turnover (Fig. 3g).

Together, these results show that Arg promotes cell retraction and redistribution of cell-matrix adhesions in response to thrombin, and that Arg depletion protects against these events.

\section{Arg promotes endothelial monolayer disruption in response to barrier-disruptive agents}

To examine the effects of Arg on monolayer integrity in response to barrier-disruptive agents, HUVEC monolayers were stimulated with thrombin for up to $30 \mathrm{~min}$. Thrombin induced pronounced AJ dissociation and intercellular gap formation in control HUVECs (indicated by arrows), which was reduced by Arg depletion (Fig. 4a and data not shown). Of note, Arg depletion predominantly reduced gap size, while gap number was less affected (Fig. 4b, c). Similar to what we observed in non-stimulated conditions (Fig. 2f), VE-cadherin internalization was also significantly decreased in Arg-depleted cells after stimulation with thrombin (Fig. 4d).

We then tested barrier function of HUVEC monolayers using Electrical Cell-substrate Impedance Sensing (ECIS). Downregulation of Arg slightly enhanced basal barrier function, and profoundly attenuated endothelial barrier disruption by thrombin (Fig. $4 \mathrm{e}-\mathrm{g}$ ), in line with the observed reduction in intercellular gap formation (Fig. $4 \mathrm{a}-\mathrm{c}$ ). Similar results were obtained using histamine (Fig. 4h). Moreover, Arg depletion also improved barrier function in human pulmonary microvascular endothelial cells (Fig. 4i). These results indicate that Arg facilitates endothelial barrier disruption in response to a variety of barrier-disruptive factors, and in endothelial cells derived from different vascular beds.

Together, these data show that Arg promotes the disassembly of AJs and intercellular gap formation, thus disrupting endothelial barrier function, and indicate that Arg inhibition can protect against endothelial barrier disruption.

\section{Arg is activated during inflammation in the endothelium and promotes inflammation-induced vascular leak in vivo}

We next investigated whether Arg contributes to vascular leak in vivo. $\mathrm{Arg}^{-1-}$ mice were apparently healthy and did not show signs of edema or spontaneous bleeding (data not shown). To evaluate the role of Arg in inflammation-induced pulmonary vascular leak, WT and $\mathrm{Arg}^{-/-}$mice were exposed to intratracheal instillation of LPS for $18 \mathrm{~h}$, thus mimicking acute lung injury. Vascular leak was measured by injection of Evans Blue (EB) one hr before sacrifice, and the immune response was measured by analyzing expression levels of inflammatory genes by qPCR. In the absence of LPS, no significant differences in EB accumulation were observed between WT and $\mathrm{Arg}^{-/}$mice (Fig. 5a). In contrast, instillation of LPS resulted in an increase in pulmonary vascular leak in WT mice, which was significantly reduced in $\mathrm{Arg}^{-/-}$mice (Fig. 5a). We observed a comparable immune response in $\mathrm{Arg}^{-/-}$mice versus WT mice upon LPS exposure, as measured by markers of macrophage activation and cytokine production (Fig. 5b, c). In addition, there was no significant difference in the numbers of extravasated cells found in the broncho-alveolar lavage fluid from WT versus $\mathrm{Arg}^{-1-}$ mice (Suppl. Fig. S4b).

Together, these results indicate that Arg depletion protects against inflammation-induced endothelial barrier disruption, while the inflammatory response and leukocyte extravasation occur normally.

In WT mice, vascular leak was paralleled by phosphorylation of CrkL at Y207, a well-known substrate of Abl kinases [41] (Fig. 5d, e). In contrast, strongly reduced LPSinduced CrkL phosphorylation at this residue was observed in $\mathrm{Arg}^{-/-}$mice, indicating that Arg is activated in the lung during inflammatory vascular leak (Fig. 5d, e). While it is possible that this result reflects the combined deletion of Arg in multiple cell types, we also investigated whether Argdependent (Y207)CrkL phosphorylation occurs in response to a barrier-disruptive stimulus in HUVECs. Indeed, thrombin stimulation caused an increase in the levels of $\mathrm{p}(\mathrm{Y} 207) \mathrm{CrkL}$, which was reduced by Arg depletion (Suppl. Fig. S4c). As a proof of concept of Arg activation in clinical disease associated with vascular leak, we performed immunohistochemistry for phosphorylation of CrkL on lung slices from critically ill, septic versus non-septic patients. Intriguingly, positive staining for $\mathrm{p}$ (Y207)CrkL was found more often in the lungs of septic patients than in non-septic patients, predominantly in the endothelium of microvessels, suggesting that Arg activation in lung endothelium occurs during sepsis (Fig. 5f, Suppl. Table S1, Suppl. Fig. S5). Finally, we also tested the effects of Arg on vascular endothelial growth factor (VEGF)-induced vascular leak. For this purpose, WT and $\mathrm{Arg}^{-/-}$mice were injected with VEGF or saline in the back skin after systemic administration of EB. Importantly, $\mathrm{Arg}^{-1-}$ mice were protected against VEGF-induced vascular leak in the skin, while no differences between WT and $\mathrm{Arg}^{-/-}$mice were observed upon saline injection (Fig. 5g, h).

Together, these data indicate that Arg is a regulator of endothelial barrier disruption and vascular leak in vivo, and determines the endothelial response to a variety of barrierdisruptive stimuli.

\section{Discussion}

Here, we identify the Abelson-family kinase Arg/Abl2 as a key regulator of endothelial barrier function. We show that knockdown of Arg expression in endothelial cells 

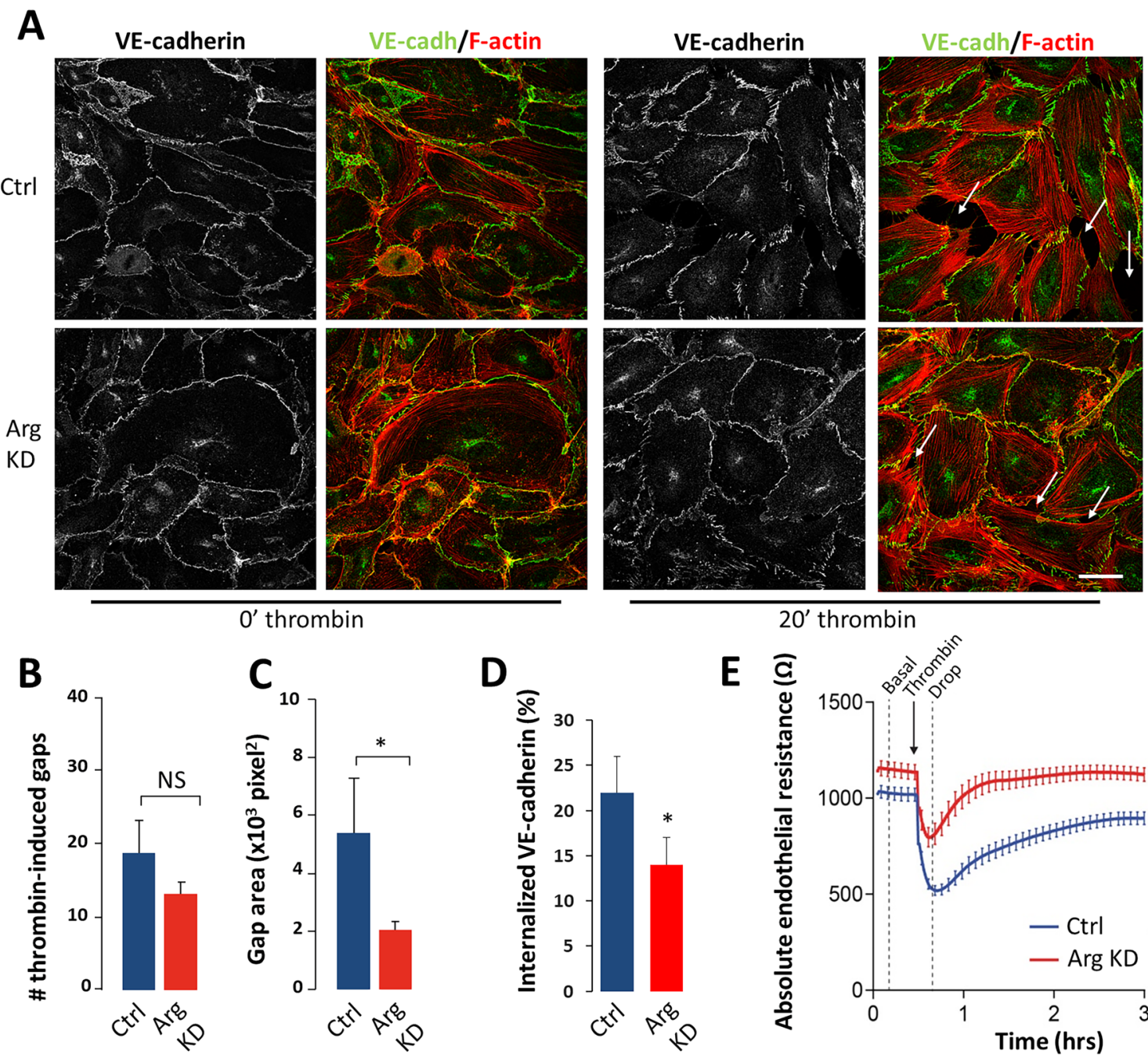

$0^{\prime}$ thrombin
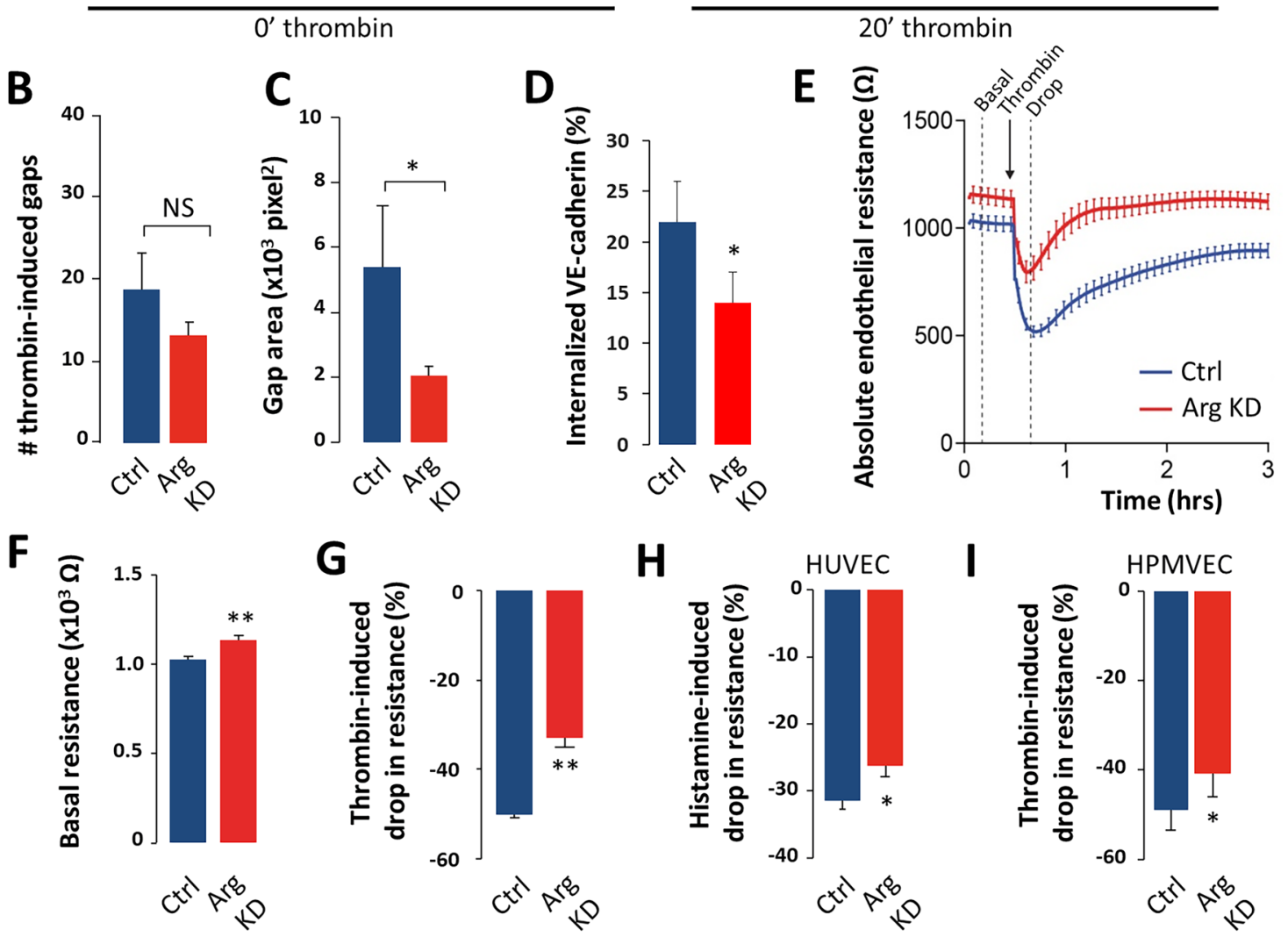

Fig. 4 Arg promotes endothelial barrier disruption in vitro. a Representative confocal images of VE-cadherin (green) and F-actin (red), showing AJ morphology in HUVEC monolayers in the absence and the presence of thrombin. Bar, $40 \mu \mathrm{m}$. Arrows indicate intercellular gaps. b Analysis of thrombin-induced intercellular gap number and (c) intercellular gap size. Mean \pm SEM of 2-3 images/donor, performed in HUVECs from 3 donors. d Internalization of VE-cadherin in HUVEC monolayers stimulated with thrombin. e Electrical resistance of confluent HUVEC monolayers before (basal) and after

thrombin addition, determined by ECIS. Quantification of $\mathbf{f}$ basal resistance and $\mathbf{g}$ thrombin-induced drop in resistance (normalized for differences in basal barrier function), as determined by ECIS. Mean + SEM of $n=19$ experiments with HUVECs from different donors. h Quantification of histamine-induced drop in endothelial resistance in HUVECs. Mean \pm SEM of $n=4$ experiments with cells from different donors. i Quantification of thrombin-induced drop in resistance in HPMVECs. Mean \pm SEM of $n=4$ experiments with cells from different donors. NS, not significant; $* \mathrm{P}<0.05 ; * * \mathrm{P}<0.01$ 
A

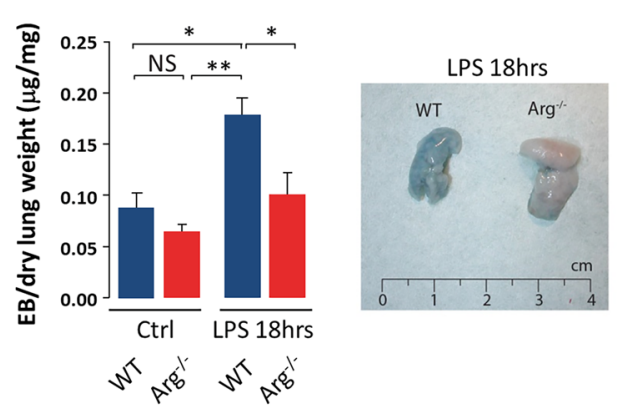

B

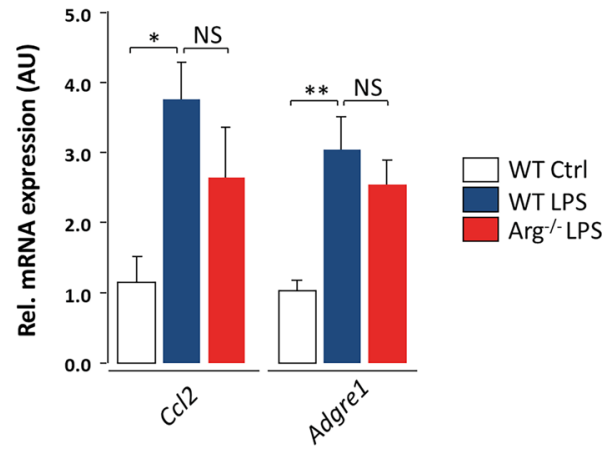

C

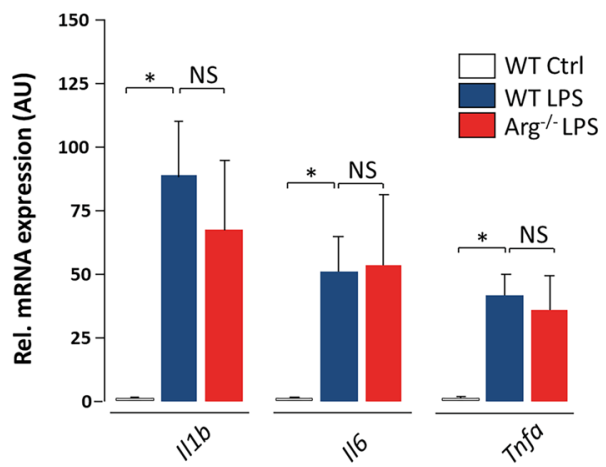

D

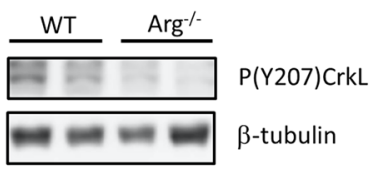

$\mathbf{F}$
$\mathbf{E}$

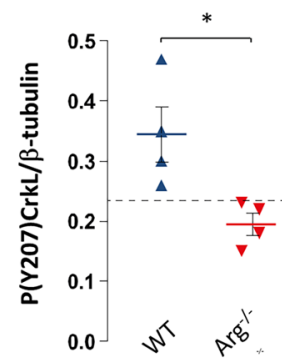

$\mathbf{G}$

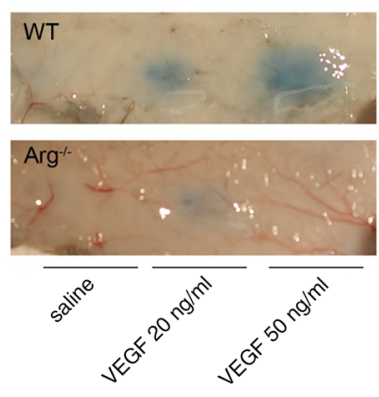

H

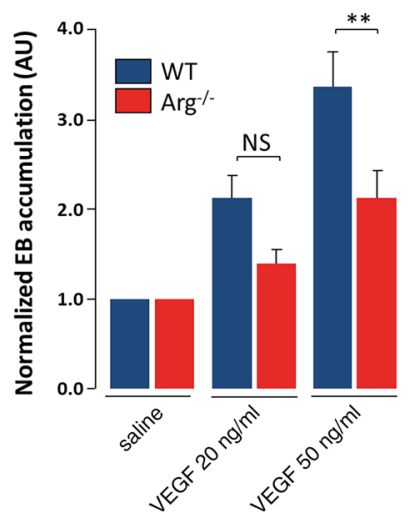

Fig. 5 Arg promotes inflammatory vascular leak in vivo. a Quantification of extravasated Evans Blue in the lungs of untreated and LPStreated mice, normalized for dry lung weight (left). Mean + SEM of $n=4-6$ mice per group. Macroscopic images show LPS-induced Evans Blue leakage in the lungs after $18 \mathrm{~h}$ (right). b mRNA levels of macrophage activation markers in lung lysates of WT versus $\mathrm{Arg}^{-/-}$ mice exposed to intra-tracheal LPS. c mRNA levels of inflammatory cytokine expression in lung lysates of WT versus $\mathrm{Arg}^{-/-}$mice exposed to intra-tracheal LPS. d Representative Western blot and (e) quantification $(n=4)$ of $\mathrm{P}(\mathrm{Y} 207) \mathrm{CrkL}$, normalized to $\beta$-tubulin, in lung tissue of WT and $\mathrm{Arg}^{-/-}$mice. $\mathbf{f}$ Representative immunohisto- chemistry images using an antibody against $\mathrm{p}(\mathrm{Y} 207) \mathrm{CrkL}$ in paraffin lung slices of 5 septic versus 4 non-septic patients. Brown staining indicates $\mathrm{p}(\mathrm{Y} 207) \mathrm{CrkL}$ (predominantly observed in the endothelial layer), blue staining indicates hematoxillin. Bars, $25 \mu \mathrm{m}$. g Macroscopic images of Evans Blue leakage in the skin after intra-cutaneous administration of PBS or VEGF in WT versus $\mathrm{Arg}^{-/-}$mice. $\mathbf{h}$ Quantification of VEGF-induced Evans Blue accumulation in the skin, normalized to that in the presence of PBS only. Means +SEM of $n=4$ mice per group. $A U$, arbitrary units; $N S$, not significant; $* \mathrm{P}<0.05$; $* * \mathrm{P}<0.01$

Arg depletion enhanced the number of integrin-based cell-matrix adhesions, in particular at the cell periphery, in line with previous observations using imatinib [27, 32]. It has been shown previously that Arg overexpression can induce phosphorylation of the NPXY motif in the integrin $\beta 1$ cytoplasmic tail [42]. However, we only detected Y783 phosphorylation in the presence of phosphatase inhibitors, indicating that it is probably weak and/or transient. More acute lung injury. 


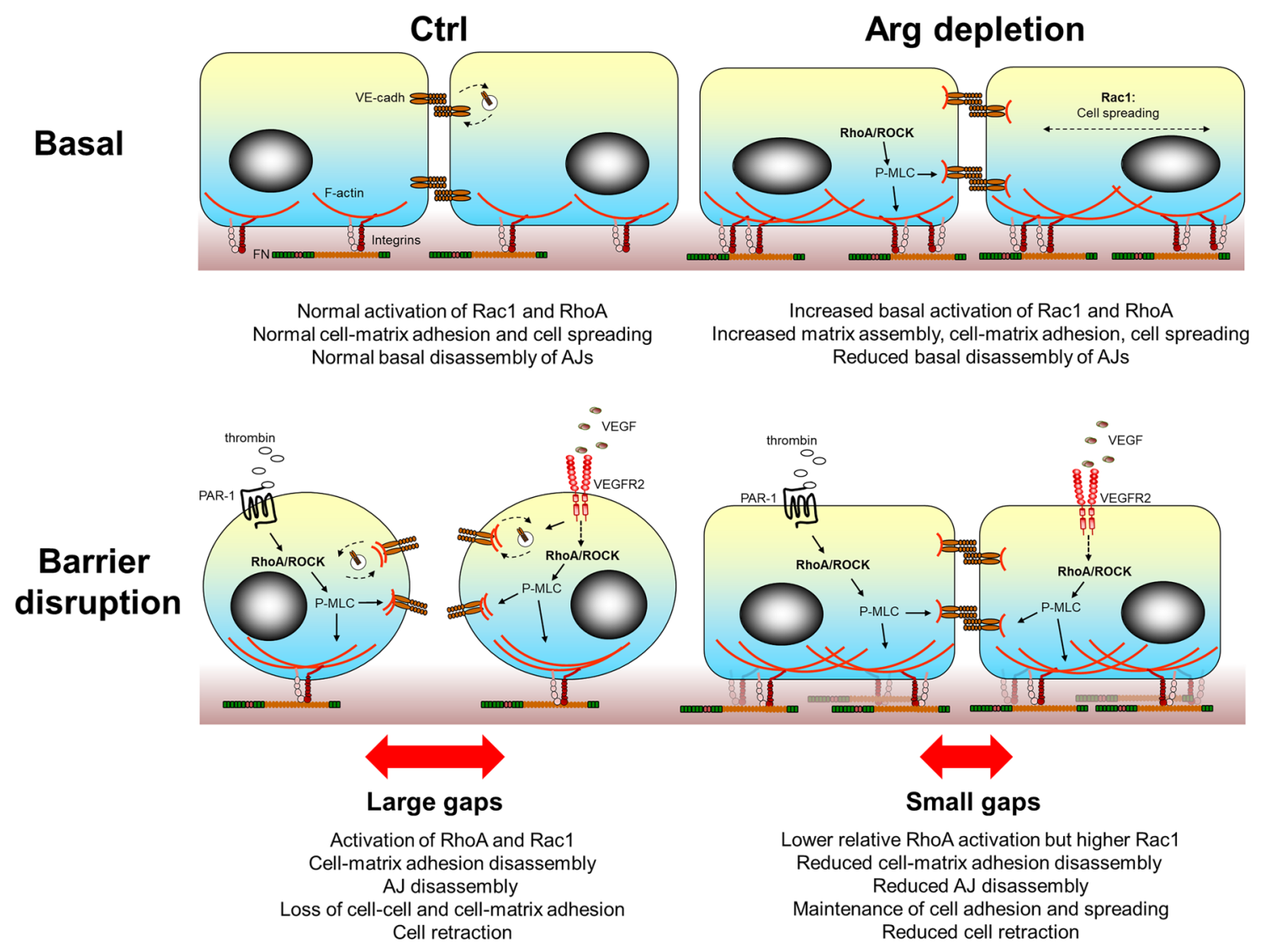

Fig. 6 Model summarizing the results described in this study. In resting endothelium, Arg depletion leads to increased basal activation of both RhoA and Rac1, thus increasing matrix assembly and cellmatrix adhesion and spreading, while the basal disassembly of AJs is reduced. Upon stimulation with a barrier-disruptive agent (thrombin, histamine and to a lesser extent VEGF), increased RhoA activation

importantly, Y783 phosphorylation was not abrogated by Arg depletion, suggesting that Arg is not required for this event in endothelial cells. Moreover, integrin activation and internalization, which are regulated by this motif, were normal in Arg-depleted HUVECs. The observed increase in cell-matrix adhesions is most likely due to the strong increase in RhoA activity upon knockdown of Arg expression. The RhoA/Rho kinase pathway enhances MLC phosphorylation by reducing its dephosphorylation, and the resulting cytoskeletal contractility promotes the stability of cell-matrix adhesions including FAs and FBs, as well as the associated integrin $\alpha 5 \beta 1$-dependent organization of FN into fibrils [48]. Indeed, we observed increased basal MLC phosphorylation and FN fibrillogenesis, as well as decreased FB turnover in Arg-depleted cells. Our findings are in line with a previous study in fibroblasts, in which it was found that Arg phosphorylates p190 RhoGAP, which inhibits Rho activity [39]. Intriguingly, increased cytoskeletal contractility can disrupt cell-cell junctions, and although leads to cytoskeletal contractility-mediated loss of cell-cell adhesion and cell retraction in control cells. In contrast, RhoA activation and contractility in Arg-depleted cells are balanced by higher Rac activation, and lower AJ disassembly, thus preserving cell-cell and cellmatrix adhesion and counteracting retraction.

the 'jagged' appearance of VE-cadherin in the absence of Arg suggests that there is indeed increased tension on AJs, it did not perturb monolayer integrity and barrier function. Moreover, the increase in contractility did not abolish cell spreading, which was in fact enhanced. The increase in cell spreading is likely due to the effects of the well-established Arg substrate CrkL, which stimulates Rac1 activation and cell spreading but whose phosphorylation at Y207 by Abl tyrosine kinases negatively regulates these events [41, 49, 50]. Consistent with this hypothesis is that we observed reduced $\mathrm{p}(\mathrm{Y} 207) \mathrm{CrkL}$ in HUVECs upon Arg depletion, while Rac1 activity was increased. There is ample evidence that Rac1 counteracts Rho-dependent contractility, and that regulation of cell size and cell spreading by Rac1 promotes the stability of AJs, thus preserving endothelial barrier function $[7,8,10]$. Furthermore, Rac1-stimulated cell spreading enhances the formation and stability of peripheral cell-matrix adhesions, which in conjunction with cell-cell junctions maintain the spread endothelial cell shape required 
for an intact monolayer, thus forming a tethering force to prevent cell retraction. Additionally, Arg may also determine the localization of active RhoA and/or phosphorylated MLC. While the results presented here show effects of Arg on global RhoA activity, future studies should address how Arg affects local activation of different subcellular RhoA pools, for instance using a RhoA biosensor [51]. Interestingly, we observed phosphorylated MLC predominantly close to tricellular cell-cell junctions under basal conditions, while thrombin stimulated increased phospho-MLC in the cell periphery and its association with circumferential actin bundles, consistent with earlier observations that phosphorylated MLC interacts with the cortical actin ring and regulates cell-cell junction integrity $[52,53]$. Of note, in a previous study in epithelial cells, the combined knockdown of both c-Abl and Arg enhanced the activity of RhoA but not that of Rac1, and in this system the increased cytoskeletal tension did result in the disruption of E-cadherin-based AJs [33]. While these data further underscore the importance of Rac1 activation to counteract RhoA-dependent contractility in the control of cell-cell junctions, it remains to be established whether there are cell-type-specific differences in Rac1 regulation by Abelson kinases, or whether this is differentially regulated by c-Abl and Arg.

Importantly, we show that in the presence of $\mathrm{c}-\mathrm{Abl}$ expression, the depletion of Arg is sufficient to preserve the barrier function of the endothelium, even after challenge with barrier-disruptive agents. Upon activation of RhoA with thrombin, the relative increase in RhoA activity and MLC phosphorylation were smaller in Arg-depleted cells, probably because the basal levels are already almost maximal. Furthermore, thrombin induced less cell retraction and intercellular gap formation in Arg-depleted cells, thus rendering the cells more refractory against barrier disruption. Together, these data suggest that Arg depletion reduces the dynamic behavior of cell-cell and cell-matrix adhesions and improves anchorage of the plasma membrane at the cell periphery (Fig. 6). The dynamic regulation of peripheral cell adhesions contributes to cell retraction and gap formation during endothelial exposure to barrier-disruptive agents, as observed in inflammation, hence anchoring of the cell periphery in Arg-depleted endothelial cells protects against barrier disruption.

Because Arg knockout mice are healthy and show no obvious defects, long-term absence of Arg is well-tolerated in vivo. Importantly, induction of inflammatory markers and cell counts in the broncho-alveolar lavage fluid were comparable in $\mathrm{Arg}^{-/-}$mice versus WT mice upon LPS exposure, corroborating previous reports that vascular leak and leukocyte extravasation are separately regulated processes $[51,54]$. These findings indicate that Arg inhibition can be harbored as treatment strategy to target vascular leakage, while leaving the immune response intact [55]. Finally, we also observed that Arg deficiency inhibited VEGF-induced vascular leak in the skin. While Arg may regulate the response to VEGF at several levels, which will be important to analyze in more detail in future studies, this finding may suggest that Arg is central to vascular leak induced by a variety of different factors. This is further suggested by our observations that Arg knockdown protected against barrier disruption induced by both thrombin and histamine in vitro. Therefore, we propose that Arg is a central and non-redundant regulator of endothelial barrier disruption in a variety of conditions and that inhibition of Arg is a suitable strategy for the treatment of clinical syndromes characterized by vascular leak, including sepsis, ARDS, and COVID-19. This is of utmost importance for critically ill patients with respiratory failure as a result of pulmonary edema, because currently no drugs are available to reverse endothelial barrier disruption under these conditions. We and others have previously shown that pharmacological inhibition of Arg by imatinib protects against leakage-induced pulmonary edema in preclinical studies [27], as well as in patients [28, 29, 31]. Moreover, a clinical trial has recently been completed by us (EudraCT \# 2020-001236-10), to establish whether imatinib also protects against respiratory failure in patients suffering from COVID-19.

Of note, imatinib inhibits c-Abl in addition to Arg, which is important because c-Abl also promotes endothelial barrier disruption [35-38]. Whereas previous studies have demonstrated the effect of c-Abl knockout [34-36] and have addressed the redundant roles of $\mathrm{c}-\mathrm{Abl}$ and $\mathrm{Arg}$ in endothelial barrier regulation [35], our study for the first time points towards a specific and non-redundant function of Arg in vivo. Together with the previously mentioned in vivo studies [34-36], it becomes apparent that while Arg and c-Abl are both involved in endothelial barrier regulation, they probably have complementary and distinct functions. Future studies are required to elucidate the unique roles of $\mathrm{c}-\mathrm{Abl}$ and Arg in barrier regulation. Because imatinib also inhibits additional tyrosine kinases, including the platelet derived growth factor receptors, development of second-generation inhibitors with increased specificity towards Abl/Arg would be preferred. Other tyrosine kinase inhibitors exist with overlapping and unique specificities, and we have recently characterized the effects of bosutinib on endothelial barrier function [56]. Bosutinib inhibits Arg as well as MAP4K4, which phosphorylates ERM proteins and thereby regulates endothelial cell adhesion [56, 57]. Thus, multiple kinases work in concert to effectuate the dynamics of endothelial cell adhesions, and it will be important in the future to further delineate the effects of each for the optimal treatment of vascular leak. 


\section{Materials and methods}

\section{Antibodies, plasmids and other materials}

The following antibodies were used: anti-c-Abl (\#2862), anti- $\beta$-tubulin (\#2128), anti-pSer19 myosin light chain (\#3671), anti-CrkL (\#3182), and anti-p(Y207)CrkL (\#3181) from Cell Signaling Technologies, anti-VEcadherin (clone C19 from Santa Cruz and clone 55-7H1 from BD Pharmingen), anti- $\beta$-catenin (Sigma Aldrich), anti- $\alpha 5$ integrin (clone VC5 from BD Pharmingen; clone MAB1999 from Chemicon), anti-Arg (NBP1-18875 from Novus Biologicals), anti-integrin $\beta 1$ (clone 9EG7 from BD Pharmingen; clone TS2/16 from the Developmental Studies Hybridoma Bank; clone P5D2 from Abcam; clone EP974(2)Y against phosphorylated Y783 from Abcam), actin (clone AC-40) and phosphotyrosines (clone 4G10 from Sigma-Aldrich), FN (clone 10), Rac1 (clone 102), and paxillin (clone 165) were from Transduction laboratories, RhoA (clone 67B9 from Cell Signaling). GFPtensin-1 was a kind gift from Dr. K. Yamada (National Institute of Health, Bethesda, MD), Rhotekin-RBD and PAK-CRIB peptides were home-made. Phalloidin was from Thermo Fischer, puromycin, human thrombin, human $\mathrm{FN}, \mathrm{Na} 3 \mathrm{VO} 4$, and $E$. coli-derived LPS were purchased from Sigma-Aldrich, VEGF from Invitrogen, and Col-I was from Vitrogen. TRITC-, FITC-, and Cy5-conjugated secondary antibodies, and DAPI were from Molecular Probes, Fugene was from Promega, and HRP-conjugated secondary antibodies were from Amersham. Primers were purchased from Integrated DNA Technologies, Inc. (sequences shown in Supplementary Table S2).

\section{In vivo measurement of vascular leakage}

Arg knockout mice with mixed $129 / \mathrm{SvJ} \times \mathrm{C} 57 \mathrm{BL} / 6 \mathrm{~J}$ background were kindly provided by Dr. A. Koleske (Yale University, New Haven, Connecticut). Heterozygous mice were bred to obtain homozygous knockout mice and wild-type littermates for experiments. Genotyping was performed with RT-PCR (primer sequences shown in Supplementary Table S2). For experiments, 10-24 weeks old mice (20-40 gr) were used, male and female in equal numbers per group.

For measurement of vascular leakage in the skin, mice were anesthetized by intraperitoneal injection with fentanyl, midazolam and acepromazine [58]. EB dye $(0.5 \%$ in PBS, $150 \mu \mathrm{L}$ ) was injected via the tail vein. Mice were shaved after $30 \mathrm{~min}$, and saline or VEGF (20 or $50 \mathrm{ng}$, dissolved in saline) was injected intra-dermally in the back skin. Mice were sacrificed $30 \mathrm{~min}$ later and the back skin was isolated. Pictures were taken and circular skin patches $(\varnothing 8 \mathrm{~mm})$ from the injection sites were incubated in formamide for $48 \mathrm{~h}$. Concentrations of EB and hemoglobin were measured spectrophotometrically at 610 and $740 \mathrm{~nm}$, respectively.

For measurement of vascular leakage in the lungs, mice were anesthetized with isoflurane $(4 \% \mathrm{v} / \mathrm{v}$ in air during induction and $1.5 \%-2 \%$ maintenance) and oxygen $0.5 \mathrm{~L} / \mathrm{min}$. The trachea was exposed by small incision of the skin, and oro-tracheal canulation was performed under view, followed by intra-tracheal administration of LPS $(2.5 \mathrm{mg} / \mathrm{kg})$ dissolved in $50 \mu \mathrm{L}$ saline, or saline only. Mice were kept upright and mildly agitated to ensure that LPS or saline reached peripheral parts of the lungs. After $17 \mathrm{~h}$, mice received 100 $\mu \mathrm{L} 1 \% \mathrm{~EB}$ via tail vein injection, which was allowed to circulate for $1 \mathrm{~h}$, after which mice were sacrificed under anesthesia. EB was extracted from the right lung by incubating in $300 \mu \mathrm{L}$ formamide at $55^{\circ} \mathrm{C}$. After $48 \mathrm{~h}$ the lungs were removed; the remaining formamide was centrifuged $(13,500$ $\mathrm{rpm}$ for $5 \mathrm{~min}$ ) and analyzed spectrophotometrically. The corrected EB absorbance was calculated by the following formula: OD610-[1.426 × OD740 + 0.03], and compared to an EB standard. The lungs were air-dried at $90{ }^{\circ} \mathrm{C}$ to determine dry weight, and vascular leak was represented as $\mathrm{EB} / \mathrm{dry}$ lung weight $(\mu \mathrm{g} / \mathrm{mg})$. The left lung was snap-frozen for tissue analysis. All animal experiments were approved by the local ethical committee for animal welfare, according to national and international guidelines.

\section{Cell culture, transfections, lentiviral transduction, and RNA interference}

Primary HUVECs pooled from 3 to 5 individual donors were purchased from Lonza (C2519A) and were cultured in endothelial growth medium-2 (Promocell, C-22011), supplemented with $2 \mathrm{mM} \mathrm{L}$-glutamine (Sigma-Aldrich) and $1 \mathrm{U} /$ $\mathrm{ml}$ penicillin/streptomycin (Sigma-Aldrich). HUVECs were used between passages 3 and 6 and routinely passaged on cell culture flasks coated with $0.1 \%(\mathrm{w} / \mathrm{v})$ gelatin (SigmaAldrich). Human pulmonary microvascular endothelial cells were freshly isolated and cultured as described before [59]. Human embryonic kidney (HEK) 293T cells (ATCC, CRL-3216) were maintained in Dulbecco's modified Eagle medium (DMEM) (Thermo Fisher Scientific) containing 4.5 g/l D-glucose, $2 \mathrm{mM}$ L-glutamine, 10\% (v/v) fetal bovine serum (Bodinco), $1 \mathrm{mM}$ sodium pyruvate (Thermo Fisher Scientific), and $1 \mathrm{U} / \mathrm{ml}$ penicillin/streptomycin. All cells were maintained at $37^{\circ} \mathrm{C}$ in a humidified atmosphere containing $5 \% \mathrm{CO} 2$.

For RNA interference we used shRNAs cloned into pLKO.1 (sequences shown in Supplementary Table S3) from the TRC Mission Library (a generous gift from Roderick Beijersbergen, Robotics and Screening Center, Netherlands 
Cancer Institute, Amsterdam). To produce lentiviral particles containing shRNAs, HEK293T cells were transfected using TransIT-LT1 transfection reagent (Mirus Bio) according to the manufacturer's protocol. Supernatant was harvested 48 and $72 \mathrm{~h}$ after transfection, centrifuged, filtered over a $0.45 \mu \mathrm{m}$ pore filter, aliquoted and stored at $-80{ }^{\circ} \mathrm{C}$. HUVECs were lentivirally transduced with either a pool of shRNAs, or with a scrambled sequence in pLKO.1 as a control. Positive cells were selected during 3 days using $1 \mu \mathrm{g} / \mathrm{ml}$ puromycin. Alternatively, siRNAs were obtained from Dharmacon (Waltham, MA) (sequences shown in Supplementary Table S3), and transiently transfected into HUVECs using DharmaFECT transfection reagent Type 1 (Dharmacon) according to the manufacturer's instructions. Cells received fresh medium $24 \mathrm{~h}$ later, and experiments were performed $48 \mathrm{~h}$ after transfection. Knockdown efficiency was evaluated in each experiment, either by Western blotting or by qPCR analysis, and similar results were obtained using siRNAs or shRNAs (data not shown).

Transient transfections of GFP-tensin-1 were performed by electroporation using the Amaxa nucleofector as described previously [26].

\section{Flow cytometry}

HUVECs were treated as indicated, detached with trypsin, and washed twice in PBS containing 2\% FCS. Cells were then stained with primary antibodies for $1 \mathrm{~h}$ on ice, washed twice, followed by incubation with secondary antibodies for $45 \mathrm{~min}$ on ice and washed twice. Cells were analyzed on a Canto-II flow cytometer (BD Immunocytometry Systems) equipped with FACSDiva software.

\section{Cell lysis and western blotting}

Cells were washed in ice-cold PBS and lysed on ice in RIPA buffer (25 mM Tris/HCl pH 7.6, $150 \mathrm{mM} \mathrm{NaCl}, 1 \% \mathrm{NP}-40$, $0.5 \%$ sodium deoxycholate, $0.1 \%$ SDS), supplemented with protease inhibitors and phosphatase inhibitors (SigmaAldrich). Cell lysates were centrifuged at $13,000 \times g$, heated at $95{ }^{\circ} \mathrm{C}$ in SDS sample buffer $(50 \mathrm{mM}$ Tris- $\mathrm{HCl} \mathrm{pH}$ 6.8, $2 \%$ SDS, $10 \%$ glycerol, $1 \% \beta$-mercaptoethanol, $12.5 \mathrm{mM}$ EDTA, $0.02 \%$ bromophenol blue), and proteins were resolved by SDS-PAGE, after which they were transferred to PVDF membranes (Millipore).

For protein analysis of mouse organ tissue, organs were snap-frozen after sacrificing the animal. Snap-frozen tissue was processed to tissue lysates by mincing $50 \mathrm{mg}$ of snapfrozen tissue in $500 \mu \mathrm{L}$ of RIPA buffer with phosphatase inhibitors and protease inhibitors. Protein concentrations were adjusted to $2 \mu \mathrm{g} / \mu \mathrm{L}$ using Bradford reagents, resolved by SDS-PAGE, and transferred to PVDF membranes for Western blotting.
Aspecific binding was blocked using 5\% (w/v) milk (Campina) in TBST (150 mM NaCl, $10 \mathrm{mM}$ Tris, $0.1 \%$ Tween 20, pH 8.0) for $30 \mathrm{~min}$, followed by incubation with primary antibodies $\left(\mathrm{o} / \mathrm{n}\right.$ at $\left.4{ }^{\circ} \mathrm{C}\right)$ and with secondary antibodies for $1 \mathrm{~h}$ at RT. Membranes were washed $3 \times$ with TBST after each step. Proteins were visualized using ECL reagent (Thermo Fisher Scientific) or the SuperSignal system (Pierce Chemical Co.), on light-sensitive films (Fuji Film) using a film processor (Konica Minolta, SRX-101A). Where indicated, quantification of band intensity was performed by densitometry using Fiji/ImageJ. Band intensity of the protein of interest was corrected to that of the corresponding loading control ( $\beta$-tubulin or vinculin).

\section{Rac1 and RhoA activity assays}

Rac1 and RhoA assays were performed as previously described [60]. Briefly, cells were seeded in $10 \mathrm{~cm}$ dishes coated with FN ( $5 \mu \mathrm{g} / \mathrm{ml})$ at 70-80\% confluency. HUVECs were stimulated with thrombin $(1 \mathrm{U} / \mathrm{ml}$ in serum-free medium) the next day for the indicated time-points and then lysed. All cells were washed with ice-cold PBS prior to lysis in buffer containing $25 \mathrm{mM}$ Tris- $\mathrm{HCl} \mathrm{pH} \mathrm{7.2,150}$ $\mathrm{mM} \mathrm{NaCl}, 10 \mathrm{mM} \mathrm{MgCl} 2,1 \% \mathrm{NP}-40,5 \%$ glycerol, and protease inhibitors. Lysates were centrifuged for $5 \mathrm{~min}$, $14000 \times g$ at $4^{\circ} \mathrm{C}$, and incubated with bacterially produced GST-Rhotekin-RBD beads (RhoA activation assay) for $\geq 1$ $\mathrm{h}$ at $4{ }^{\circ} \mathrm{C}$. After incubation, samples were centrifuged for 20 sec, $5000 \times g$ at $4^{\circ} \mathrm{C}$, GST-Rhotekin-RBD beads were placed on ice, and supernatants were incubated for $30 \mathrm{~min}$ with 30 $\mu \mathrm{g}$ of a biotinylated PAK1-CRIB peptide (Rac1 activation assay) coupled to streptavidin agarose beads. Subsequently, all beads were washed 5 times with lysis buffer, boiled in SDS sample buffer, and analyzed by Western blotting. Bands of pulldowns as well as total cell lysates were quantified by densitometry using Fiji/ImageJ. Results are expressed as the ratio active/total GTPase.

\section{Internalization assays and capture-ELISA}

Internalization assays were performed essentially as described earlier with some modifications [26, 45-47]. Briefly, cells were treated as indicated, transferred to ice, washed twice in ice-cold PBS and surface-labeled at 4 ${ }^{\circ} \mathrm{C}$ with $133 \mu \mathrm{g} / \mathrm{ml} \mathrm{NHS}-\mathrm{SS}-$ biotin for $60 \mathrm{~min}$. Cells were washed 3 times with ice-cold PBS before transfer to serumfree medium at $37^{\circ} \mathrm{C}$ in the presence of $0.6 \mathrm{mM}$ primaquine (Sigma-Aldrich) to block recycling, after which they were washed twice with ice-cold PBS. Remaining cell-surface biotin was removed by reduction with $3.89 \mathrm{mg} / \mathrm{ml} \mathrm{MesNa}$

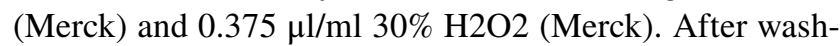
ing in PBS, the reduction was quenched with $3.45 \mathrm{mg} / \mathrm{ml}$ Iodoacetamide (Sigma-Aldrich) for $60 \mathrm{~min}$ at $4 \mathrm{C}$, whereafter 
the cells were lysed in $200 \mathrm{mM} \mathrm{NaCl}, 75 \mathrm{mM}$ Tris, $7.5 \mathrm{mM}$ EDTA, 7.5 mM EGTA, 2.25\% NP-40, $100 \mu \mathrm{M}$ Na3VO3, and protease inhibitor cocktail (Sigma-Aldrich).

Maxisorb 96-well plates (Life Technologies) were coated $\mathrm{o} / \mathrm{n}$ with $5 \mu \mathrm{g} / \mathrm{ml}$ anti-integrin $\alpha 5$ (Clone VC5), anti-integrin $\beta 1$ (Clone P5D2), or anti-VE-cadherin (Clone 55-7H1) antibodies in $0.05 \mathrm{M} \mathrm{Na} 2 \mathrm{CO} 3, \mathrm{pH} 9.6$ at $4{ }^{\circ} \mathrm{C}$, and then blocked for $1 \mathrm{~h}$ at RT in PBST with 5\% BSA. Plates were incubated with cell lysates $\left(\mathrm{o} / \mathrm{n}\right.$ at $\left.4^{\circ} \mathrm{C}\right)$, washed with PBST, and incubated with HRP-streptavidin in PBST with $0.1 \%$ BSA for $1 \mathrm{hr}$ at $4{ }^{\circ} \mathrm{C}$. After washing, biotinylated proteins were detected with ortho-phenylenediamine (Sigma-Aldrich) in a buffer containing $25.4 \mathrm{mM} \mathrm{Na} 2 \mathrm{HPO} 4,12.3 \mathrm{mM}$ citric acid (pH 5.5) and $0.003 \% \mathrm{H} 2 \mathrm{O} 2$. The reaction was terminated with $8 \mathrm{M} \mathrm{H} 2 \mathrm{SO} 4$ and absorbance was read at $490 \mathrm{~nm}$.

\section{Live imaging of fibrillar adhesions and analysis}

HUVECs were transduced with shRNAs and selected with puromycin, whereafter they were transfected with GFP-tensin-1 and grown to confluency on Nunc Lab-Tek Chambered \#1.0 Borosilicate coverglass systems (Thermo Fisher Scientific). Cells were stimulated with thrombin the next day for the indicated time, and time-lapse sequences were recorded on a Zeiss Observer Z1 microscope (40× magnification, 60 $\mathrm{s}$ intervals). Movies were generated with Fiji/ImageJ, and the assembly and disassembly rate of fibrillar adhesions were determined using open-source software (http://gomez lab.bme.unc.edu/tools) essentially as described previously [61] with the following specifications: imaging frequency 1, detection threshold 2, min adhesion size 5, min FA phase length 10, min FAAI ratio 3 .

\section{qPCR}

For analysis of relative mRNA expression levels in transduced HUVECs, total RNA was isolated using the RNeasy kit (Qiagen) according to the manufacturer's instructions. After reverse transcription to cDNA using the SuperScript III First-Strand Synthesis System (Thermo Fisher Scientific), qPCR was performed with the SensiFAST SYBR NoROX kit (Bioline) and the indicated primers (Supplementary Table S2). The PCR was performed and analyzed either on a LightCycler PCR system (Roche) or a StepOnePlus system (Applied Biosystems). Duplicate reactions were performed for each gene.

For mRNA analysis of mouse lung tissue, lungs were snap-frozen in liquid nitrogen directly after sacrificing the animal. RNA was isolated from $30 \mathrm{mg}$ of snap-frozen lung tissue, using the RNeasy Minikit (Qiagen). Isolated mRNA was used for cDNA synthesis, using iScript reaction mix and iScript reverse transcriptase. cDNA was used for qPCR with the indicated primers (Supplementary Table S2). Reference genes for cultured endothelial cells included $G A P D H$ or $T U B B 2$, while Rps 15 was used as a reference gene for qPCR analysis of mouse tissue.

\section{Confocal microscopy and quantifications}

HUVECs on glass coverslips were treated as indicated and then fixed with 4\% paraformaldehyde (Merck) in PBS containing $1 \mathrm{mM} \mathrm{CaCl} 2$ and $0.5 \mathrm{mM} \mathrm{MgCl} 2$ (PBS++) for 10 min, and permeabilized with $0.4 \%$ Triton X-100 (SigmaAldrich) in PBS++ for $5 \mathrm{~min}$. Aspecific antibody binding was prevented by blocking with $2 \%$ BSA Fraction V (SigmaAldrich) in PBS++ for 15 min. Following incubation with the indicated primary antibodies, coverslips were washed with $0.5 \%$ BSA Fraction V in PBS++ and antibody binding was visualized using secondary antibodies. Coverslips were washed and subsequently mounted in $10 \%$ Mowiol, $2.5 \%$ Dabco, $25 \%$ glycerol, $\mathrm{pH}$ 8.5. Image acquisition was performed on a Leica SP8 confocal microscope using a $63 \times$ oil immersion objective, after which images were processed using Leica Application Suite X and Fiji/ImageJ (version $1.52 \mathrm{e})$ software.

The number of peripheral adhesions relative to the cell as a whole was quantified using Fiji/ImageJ essentially as described earlier with some modifications [39]. In short, images were converted to 8-bit grayscale, foreground/ background colors were inverted, and threshold-adjusted. Individual cell outlines were generated using the freehand selection tool. The "analyze particles" function was used to select and measure the peripheral area of fluorescence within the freehand selection. The particle areas for the peripheral selection were summed and divided by the total freehand selection area to obtain the relative density at the periphery. Individual selection densities from 20 cells were averaged to obtain the density values per cell. To quantify thrombininduced intercellular gap formation from confocal images, images were converted to binary images, in which stainingnegative areas were considered as being a gap. Gap number and size were determined using the ImageJ particle analysis tool.

Femoral artery segments (1-2 mm) were freshly isolated from sacrificed mice and mounted to glass canulae. After saline perfusion under pressure, vessels were fixed with 4\% paraformaldehyde (Sigma Aldrich) for $15 \mathrm{~min}$ at room temperature. Fixed vessel segment were cut in the length, opened and mounted to a silicon underground with tungsten wire at the four corners. Subsequently segments were washed with saline and stained with a primary antibody against VE-cadherin (rabbit, 1:200) overnight at $4{ }^{\circ} \mathrm{C}$. After washing, cells were incubated with FITC- and Cy3-labeled secondary antibodies (anti-mouse and anti-rabbit, 1:100) (Invitrogen, Paisly, United Kingdom). Finally, vessel segments were mounted on glass coverslips with Vectashield 
mounting medium, containing DAPI (Vector Laboratories Inc, Burlingham, CA). Imaging was performed as described for cultured endothelial cells.

\section{Immunohistochemistry of human lung tissue}

Paraffin-embedded lung tissue of septic and critically ill, non-septic patients obtained by autopsy was provided by the Department of Pathology of our hospital (Amsterdam University Medical Center, location VUmc, Amsterdam, The Netherlands). Paraffin slices $(5 \mu \mathrm{m})$ were de-paraffinized with xylene and incubated with $\mathrm{H}_{2} \mathrm{O}_{2}$ to block endogenous peroxidase. After antigen retrieval (TRIS/EDTA $10 \mathrm{mM}$ $\mathrm{pH} 9.0,15 \mathrm{~min}$ at $100^{\circ} \mathrm{C}$ ), slices were incubated with primary antibody against $\mathrm{p}(\mathrm{Y} 207) \mathrm{CrkL}\left(\mathrm{o} / \mathrm{n}\right.$ at $\left.4{ }^{\circ} \mathrm{C}\right)$. Detection of primary antibody was performed with Powervision ${ }^{\circledR}$ (Immunologic, Duiven, The Netherlands), according to the manufacturers protocol. After counterstaining with hematoxylin, slices were evaluated with a Leica DMRB light microscope (Leica Microsystems, Wetzlar, Germany) at 20x (air, NA 0.40) and 40x (air, NA 0.65) magnification. For imaging, a Nikon D50 digital camera (Nikon Corporation, Tokyo, Japan) was used.

\section{Endothelial barrier function measurements}

Endothelial barrier function was measured by Electrical Cell-substrate Impedance Sensing (ECIS). Confluent cells were seeded in $1: 1$ density $\left(5 \times 10^{4}\right.$ cells $\left./ \mathrm{cm}^{2}\right)$ on gelatin-coated ECIS arrays, containing 8 wells with 10 gold electrodes/well (Applied Biophysics, Troy, NY). Culture medium was renewed $24 \mathrm{~h}$ after seeding, while experiments were performed $48 \mathrm{~h}$ after seeding. For thrombin stimulation, cells were incubated in serum-free medium containing 1\% human serum albumin (HSA). After 60-90 min of preincubation, thrombin $(1 \mathrm{U} / \mathrm{ml})$ or histamine $(10 \mu \mathrm{M})$ was added to the wells.

\section{Cell adhesion and cell spreading assays}

Plates were coated with Col-I ( $3 \mu \mathrm{g} / \mathrm{ml}, 5 \mathrm{~min})$ or FN ( $5 \mu \mathrm{g} /$ $\mathrm{ml}, 90 \mathrm{~min}$ ) at $37^{\circ} \mathrm{C}$. Thereafter, they were washed twice with PBS, blocked with $2 \% \mathrm{BSA} / \mathrm{PBS}$ for $30 \mathrm{~min}$ at $37{ }^{\circ} \mathrm{C}$, and washed with PBS. For adhesion assays, subconfluent cells were trypsinized, resuspended in serum-free medium, and then seeded in 96-well plates coated with Col-I or FN at a density of $7 \times 10^{4}$ cells per well. After $10 \mathrm{~min}$ at $37^{\circ} \mathrm{C}$, non-adherent cells were washed away with PBS. The adherent cells were fixed in 4\% PFA, washed twice with $\mathrm{H} 2 \mathrm{O}$, stained for 10 min with crystal violet, washed twice with $\mathrm{H} 2 \mathrm{O}$, and then lysed in $2 \%$ SDS. Absorbance was measured at $490 \mathrm{~nm}$ on a microplate reader. Background values (binding to BSA-coated wells) were subtracted from all other values, and the number of adherent cells was normalized to that of control cells.

For cell spreading, cells were seeded in serum-free medium on 24-well plates coated with Col-I or FN, and cell spreading was allowed for $3 \mathrm{~h}$. Cells were then photographed on a Widefield CCD system using $10 \times$ and $20 \times$ dry lens objectives (Carl Zeiss MicroImaging, Inc.). Cell area and elongation index (length/width) were determined using Fiji/ ImageJ.

\section{Statistical analysis}

Statistical analysis was performed using one-way ANOVA for multiple comparisons and unpaired $t$ tests for comparisons between two conditions, unless stated otherwise. Throughout the paper, statistical significance is indicated by $*(\mathrm{P}<0.05)$ and $* *(\mathrm{P}<0.01)$.

Supplementary Information The online version contains supplementary material available at https://doi.org/10.1007/s10456-021-09781-x.

Acknowledgements We thank Tony Koleske, Ken Yamada, Arnoud Sonnenberg, and Roderick Beijersbergen for their generous gifts of reagents. We gratefully acknowledge technical support from Mark Hoogenboezem, Simon Tol, and Erik Mul (Central Facility, Sanquin Research, Amsterdam), and Ben Morris (Robotics and Screening Center, Netherlands Cancer Institute, Amsterdam). Arnoud Sonnenberg is thanked for critical reading of the manuscript.

Funding This work was supported by grants from the Netherlands Heart Foundation to G.P. van Nieuw Amerongen (\#2003T3201) and J. Aman (\#2014T064), the ESICM/ECCRN Young Investigator Award 2011 to J. Aman, and the Netherlands Organization for Scientific Research (NWO) to C. Margadant (ZonMW Veni 016.146.160).

Data availability The datasets generated during and/or analysed during the current study are available from the corresponding author on reasonable request.

\section{Compliance with ethical standards}

Conflict of interest The authors declare no conflict of interest.

Ethical approval All applicable (inter-)national and/or institutional guidelines for the care and use of animals were followed. The ethics approval statements for human subjects were provided by the Medical Ethical Committee of the Amsterdam University Medical Center. Informed consent was obtained from all subjects for the use of tissue for research purposes.

Open Access This article is licensed under a Creative Commons Attribution 4.0 International License, which permits use, sharing, adaptation, distribution and reproduction in any medium or format, as long as you give appropriate credit to the original author(s) and the source, provide a link to the Creative Commons licence, and indicate if changes were made. The images or other third party material in this article are included in the article's Creative Commons licence, unless indicated otherwise in a credit line to the material. If material is not included in the article's Creative Commons licence and your intended use is not 
permitted by statutory regulation or exceeds the permitted use, you will need to obtain permission directly from the copyright holder. To view a copy of this licence, visit http://creativecommons.org/licenses/by/4.0/.

\section{References}

1. Goldenberg N, Steinberg B, Slutsky A, Lee W (2011) Broken barriers: a new take on sepsis pathogenesis. Sci Transl Med 3:25

2. Matthay M, Ware L, Zimmerman G (2012) The acute respiratory distress syndrome. J Clin Invest 122:2731-40

3. Teuwen LA, Geldhof V, Pasut A, Carmeliet P (2020) COVID-19: the vasculature unleashed. Nat Rev Immunol 20:389-391

4. Mehta D, Malik A (2006) Signaling mechanisms regulating endothelial permeability. Physiol Rev 86:279-367

5. Vandenbroucke E, Mehta D, Minshall R, Malik AB (2008) Regulation of endothelial junctional permeability. Ann N Y Acad Sci 1123:134-145

6. Oldenburg J, de Rooij J (2014) Mechanical control of the endothelial barrier. Cell Tissue Res 355:545-555

7. Wojciak-Stothard B, Potempa S, Eichholtz T, Ridley AJ (2001) Rho and Rac but not Cdc42 regulate endothelial cell permeability. J Cell Sci 114:1343-1355

8. Beckers CM, van Hinsbergh VW, van Nieuw Amerongen GP (2010) Driving Rho GTPase activity in endothelial cells regulates barrier integrity. Thromb Haemost 103:40-55

9. Huveneers S, Oldenburg J, Spanjaard E et al (2012) Vinculin associates with endothelial VE-cadherin junctions to control forcedependent remodeling. J Cell Biol 196:641-652

10. Ratheesh A, Priya R, Yap AS (2013) Coordinating Rho and Rac: the regulation of Rho GTPase signaling and cadherin junctions. Prog Mol Biol Transl Sci 116:49-68

11. van Nieuw Amerongen G, Musters R, Eringa E et al (2008) Thrombin-induced endothelial barrier disruption in intact microvessels: role of RhoA/Rho kinase-myosin phosphatase axis. Am J Physiol Cell Physiol 294:C1234-41

12. van Nieuw Amerongen G, Natarajan K, Yin G et al (2004) GIT1 mediates thrombin signaling in endothelial cells: role in turnover of RhoA-type focal adhesions. Circ Res 94:1041-9

13. Birukova A, Smurova K, Birukov K et al (2004) Role of Rho GTPases in thrombin-induced lung vascular endothelial cells barrier dysfunction. Microvasc Res 67:64-77

14. Yamamoto H, Ehling M, Kato K et al (2015) Integrin beta1 controls VE-cadherin localization and blood vessel stability. Nat Commun 6:6429

15. Lampugnani M, Resnati M, Dejana E, Marchisio P (1991) The role of integrins in the maintenance of endothelial monolayer integrity. J Cell Biol 112:479-490

16. Wu MH, Ustinova E, Granger HJ (2001) Integrin binding to fibronectin and vitronectin maintains the barrier function of isolated porcine coronary venules. J Physiol 532:785-791

17. Pulous F, Grimsley-Myers C, Kansal S et al (2019) Talin-dependent integrin activation regulates ve-cadherin localization and endothelial cell barrier function. Circ Res 124:891-903

18. Hakanpaa L, Sipila T, Leppanen VM et al (2015) Endothelial destabilization by angiopoietin-2 via integrin beta1 activation. Nat Commun 6:5962

19. Hakanpaa L, Kiss EA, Jacquemet G et al (2018) Targeting beta1integrin inhibits vascular leakage in endotoxemia. Proc Natl Acad Sci USA 115:E6467-E6476

20. Al-Yafeai Z, Yurdagul AJ, Peretik J et al (2018) Endothelial FN (Fibronectin) deposition by $\alpha 5 \beta 1$ integrins drives atherogenic inflammation. Arter Thromb Vasc Biol 38:2601-2614
21. Feaver R, Gelfand B, Wang C et al (2010) Atheroprone hemodynamics regulate fibronectin deposition to create positive feedback that sustains endothelial inflammation. Circ Res 106:1703-11

22. Danen EH, Sonneveld P, Brakebusch C et al (2002) The fibronectin-binding integrins alpha5beta 1 and alphavbeta 3 differentially modulate RhoA-GTP loading, organization of cell matrix adhesions, and fibronectin fibrillogenesis. J Cell Biol 159:1071-1086

23. Lin GL, Cohen DM, Desai RA et al (2013) Activation of beta1 but not beta3 integrin increases cell traction forces. FEBS Lett 587:763-769

24. Mao Y, Schwarzbauer J (2005) Fibronectin fibrillogenesis, a cellmediated matrix assembly process. Matrix Biol 24:389-99

25. van der Bijl I, Nawaz K, Kazlauskaite U et al (2020) Reciprocal integrin/integrin antagonism through kindlin-2 and Rho GTPases regulates cell cohesion and collective migration. Matrix Biol 93:60-78

26. Margadant C, Kreft M, de Groot DJ et al (2012) Distinct roles of talin and kindlin in regulating integrin alpha5beta1 function and trafficking. Curr Biol 22:1554-1563

27. Aman J, van Bezu J, Damanafshan A et al (2012) Effective treatment of edema and endothelial barrier dysfunction with imatinib. Circulation 126:2728-38

28. Carnevale-Schianca F, Gallo S, Rota-Scalabrini D et al (2011) Complete resolution of life-threatening bleomycin-induced pneumonitis after treatment with imatinib mesylate in a patient with Hodgkin's lymphoma: hope for severe chemotherapy-induced toxicity? J Clin Oncol 29:e691-693

29. Overbeek M, van Nieuw AG, Boonstra A et al (2008) Possible role of imatinib in clinical pulmonary veno-occlusive disease. Eur Respir J 32:232-235

30. Rizzo A, Aman J, van Nieuw AG, Dudek S (2015) Targeting Abl kinases to regulate vascular leak during sepsis and acute respiratory distress syndrome. Arter Thromb Vasc Biol 35:1071-1079

31. Aman J, Peters M, Weenink C et al (2012) Reversal of vascular leak with imatinib. Am J Respir Crit Care Med 188:1171-1173

32. Chen Z, Lessey E, Berginski M et al (2013) Gleevec, an Abl family inhibitor, produces a profound change in cell shape and migration. PLoS One 8:e52233

33. Zandy NL, Playford M, Pendergast AM (2007) Abl tyrosine kinases regulate cell - cell adhesion through Rho GTPases. Proc Natl Acad Sci U S A 104:17686-17691

34. Chislock E, Ring C, Pendergast A (2013) Abl kinases are required for vascular function, Tie2 expression, and angiopoietin-1-mediated survival. Proc Natl Acad Sci U S A 110:12432-7

35. Chislock E, Pendergast A (2013) Abl family kinases regulate endothelial barrier function in vitro and in mice. PLoS One 8:e85231

36. Fantin A, Lampropoulou A, Senatore V et al (2017) VEGF165induced vascular permeability requires NRP1 for ABL-mediated SRC family kinase activation. J Exp Med 214:1049-1064

37. Sun Y, Hu GHZ, Minshall R (2009) Phosphorylation of caveolin-1 regulates oxidant-induced pulmonary vascular permeability via paracellular and transcellular pathways. Circ Res 105:676-685

38. Dudek SM, Chiang ET, Camp SM et al (2010) Abl tyrosine kinase phosphorylates nonmuscle mysoin light chain kinase to regulate endothelial barrier function. Mol Biol Cell 21:4042-4056

39. Peacock J, Miller A, Bradley W et al (2007) The Abl-related gene tyrosine kinase acts through p190RhoGAP to inhibit actomyosin contractility and regulate focal adhesion dynamics upon adhesion to fibronectin. Mol Biol Cell 18:3860-72

40. Bradley W, Hernandez S, Settleman J, Koleske A (2006) Integrin signaling through Arg activates p190RhoGAP by promoting its binding to $\mathrm{p} 120 \mathrm{RasGAP}$ and recruitment to the membrane. Mol Biol Cell 17:4827-36 
41. Birge RB, Kalodimos C, Inagaki F, Tanaka S (2009) Crk and CrkL adaptor proteins : networks for physiological and pathological signaling. Cell Commun Signal 23:7-13

42. Simpson M, Bradley W, Harburger D et al (2015) Direct interactions with the integrin b1 cytoplasmic tail activate the Abl2/Arg kinase. J Biol Chem 290:8360-72

43. Lapetina S, Mader CC, Machida K et al (2008) Arg interacts with cortactin to promote adhesion-dependent cell edge protrusion. $\mathrm{J}$ Cell Biol 185:503-519

44. Li R, Pendergast A (2011) Arg kinase regulates epithelial cell polarity by targeting beta1-integrin and small GTPase pathways. Curr Biol 21:1534-42

45. Roberts M, Barry S, Woods A et al (2001) PDGF-regulated rab4dependent recycling of $\alpha \mathrm{v} \beta 3$ integrin from early endosomes is necessary for cell adhesion and spreading. Curr Biol 11:1392-1402

46. Caswell PT, Chan M, Lindsay AJ et al (2008) Rab-coupling protein coordinates recycling of $\alpha 5 \beta 1$ integrin and EGFR1 to promote cell migration in 3D microenvironments. J Cell Biol 183:143-155

47. Jonker C, Galmes R, Veenendaal T et al (2018) Vps3 and Vps8 control integrin trafficking from early to recycling endosomes and regulate integrin-dependent functions. Nat Commun 9:1-12

48. Lawson CD, Burridge K (2014) The on-off relationship of Rho and Rac during integrin-mediated adhesion and cell migration. Small GTPases 5:e27958

49. Park T-J, Curran T (2014) Essential roles of Crk and CrkL in fibroblast structure and motility. Oncogene 33:5121-5132

50. Antoku S, Mayer BJ (2009) Distinct roles for Crk adaptor isoforms in actin reorganization induced by extracellular signals. J Cell Sci $122: 4228-4238$

51. Heemskerk N, Schimmel L, Oort C et al (2016) F-actin-rich contractile endothelial pores prevent vascular leakage during leukocyte diapedesis through local RhoA signalling. Nat Commun 7:10493

52. Fong LY, Ng CT, Yong YK et al (2019) Asiatic acid stabilizes cytoskeletal proteins and prevents TNF- $\alpha$-induced disorganization of cell-cell junctions in human aortic endothelial cells. Vascul Pharmacol 117:15-26
53. Birukov KG, Birukova AA, Dudek SM et al (2002) Shear stressmediated cytoskeletal remodeling and cortactin translocation in pulmonary endothelial cells. Am J Respir Cell Mol Biol 26:453-64

54. Wessel F, Winderlich M, Holm M et al (2014) Leukocyte extravasation and vascular permeability are each controlled in vivo by different tyrosine residues of VE-cadherin. Nat Immunol 15:223-30

55. Filewod NC, Lee WL (2019) Inflammation without vascular leakage. Science fiction no longer? Am J Respir Crit Care Med 200:1472-1476

56. Botros L, Pronk MCA, Juschten J et al (2020) Bosutinib prevents vascular leakage by reducing focal adhesion turnover and reinforcing junctional integrity. J Cell Sci 133:jcs240077

57. Vitorino P, Yeung S, Crow A et al (2015) MAP4K4 Regulates integrin-FERM Binding to Control Endothelial Cell Motility. Nature 519:425-430

58. Weis S, Cheresh D (2005) Pathophysiological consequences of VEGF-induced vascular permeability. Nature 437:497-504

59. van der Heijden M, van Nieuw Amerongen GP, van Bezu J et al (2011) Opposing effects of the angiopoietins on the thrombin- induced permeability of human pulmonary microvascular endothelial cells. PLoS One 6:e23448

60. Sander E, ten Klooster JP, van Delft S et al (1999) Rac downregulates Rho activity: Reciprocal balance between both GTPases determines cellular morphology and migratory behaviour. J Cell Biol 147:1009-1021

61. Berginski ME, Vitriol EA, Hahn KM, Gomez SM (2011) Highresolution quantification of focal adhesion spatiotemporal dynamics in living cells. PLoS One 6:e22025

Publisher's Note Springer Nature remains neutral with regard to jurisdictional claims in published maps and institutional affiliations. 